\title{
Mod $\ell$ representations of arithmetic fundamental groups II: A conjecture of A. J. de Jong
}

\author{
Gebhard Böckle and Chandrashekhar Khare
}

\begin{abstract}
We study deformation rings of an $n$-dimensional representation $\bar{\rho}$, defined over a finite field of characteristic $\ell$, of the arithmetic fundamental group $\pi_{1}(X)$, where $X$ is a geometrically irreducible, smooth curve over a finite field $k$ of characteristic $p(\neq \ell)$. When $\bar{\rho}$ has large image, we are able to show that the resulting rings are finite flat over $\mathbf{Z}_{\ell}$. The proof principally uses a Galois-theoretic lifting result of the authors in Part I of this two-part work, a lifting result for cuspidal mod $\ell$ forms of Ogilvie, Taylor-Wiles systems and the result of Lafforgue. This implies a conjecture of de Jong for representations of $\pi_{1}(X)$ with coefficients in power series rings over finite fields of characteristic $\ell$, that have this mod $\ell$ representation $\bar{\rho}$ as their reduction. A proof of all cases of the conjecture for $\ell>2$ follows from a result announced by Gaitsgory. The methods are different.
\end{abstract}

\section{Contents}

1 Introduction $\quad 271$

1.1 Results . . . . . . . . . . . . . . . . . . . . . 272

1.2 Sketch of proof . . . . . . . . . . . . . . . . 273

1.3 Some remarks . . . . . . . . . . . . . . . . . . . 273

2 Galois cohomology $\quad 274$

2.1 On deformations of the determinant . . . . . . . . . . . . . . . . 274

2.2 Removing local ramification . . . . . . . . . . . . . . . . . 275

2.3 On Taylor-Wiles auxiliary primes . . . . . . . . . . . . . . . 277

3 Automorphic methods 281

3.1 Automorphic forms over function fields . . . . . . . . . . . . . . . 282

3.2 Hecke algebras . . . . . . . . . . . . . . . . . . . . 283

3.3 Carayol's principle . . . . . . . . . . . . . . . . . 285

3.4 Taylor-Wiles systems _. . . . . . . . . . . . . . 286

3.5 Lowering the level $\grave{a}$ la Skinner and Wiles . . . . . . . . . . . . . . . . . 290

4 Proof of main theorems $\quad 290$

$\begin{array}{ll}\text { Appendix } & 292\end{array}$

$\begin{array}{ll}\text { References } & 293\end{array}$

\section{Introduction}

Let $X$ be a geometrically irreducible, smooth curve over a finite field $k$ of characteristic $p$ and cardinality $q$. Denote by $K$ its function field and by $\widetilde{X}$ its smooth compactification and set $S:=$ $\widetilde{X} \backslash X$. Let $\pi_{1}(X)$ denote the arithmetic fundamental group of $X$. Thus $\pi_{1}(X)$ sits in the exact

Received 17 June 2004, accepted in final form 18 October 2005.

2000 Mathematics Subject Classification 11F80, 11F70, 14H30, $11 \mathrm{R} 34$.

Keywords: automorphic forms, Galois representations, function fields.

This journal is (C) Foundation Compositio Mathematica 2006. 


\section{G. BöCKLE AND C. Khare}

sequence

$$
0 \rightarrow \pi_{1}(\bar{X}) \rightarrow \pi_{1}(X) \rightarrow G_{k} \rightarrow 0,
$$

where $\bar{X}$ is the base change of $X$ to an algebraic closure of $k$, and $G_{F}$ denotes the absolute Galois group of any field $F$. In this paper we study deformation rings of $\bmod \ell$ representations of $\pi_{1}(X)$ : fix a continuous, absolutely irreducible representation $\bar{\rho}: \pi_{1}(X) \rightarrow \mathrm{GL}_{n}(\mathbf{F})$ with $\mathbf{F}$ a finite field of characteristic $\ell \neq p$. We begin with the following conjecture of de Jong.

Conjecture 1.1 [dJo01, Conjecture 1.1]. Let $\rho: \pi_{1}(X) \rightarrow \mathrm{GL}_{n}(\mathbf{F}[[x]])$ be a continuous representation with residual representation $\bar{\rho}$. Then $\rho\left(\pi_{1}(\bar{X})\right)$ is finite.

Remark 1.2.

(i) De Jong [dJo01] proves the above for $n \leqslant 2$ by extending Drinfeld's reciprocity theorem [Dri83] to $\mathbf{F}((x))$-coefficients.

(ii) It is an important feature, observed in [dJo01, Lemma 2.12], of the representations considered in Conjecture 1.1 that the image of any inertia group (for a place in $S$ ) is finite. We will exploit this several times.

To state a reformulation of the above, we need some notation. Let $\mathcal{O}$ be the ring of integers of a finite extension of the fraction field of $W(\mathbf{F})$ inside $\overline{\mathbf{Q}}_{\ell}$, let $\bar{\rho}$ be as above and fix a lift $\eta: \pi_{1}(X) \rightarrow \mathcal{O}^{*}$ of finite order of the one-dimensional representation $\operatorname{det} \bar{\rho}$. Then in [dJo01], following [Maz89], it is explained how to attach a deformation ring $R_{X, \mathcal{O}}^{\eta}(\bar{\rho})$, or simply $R_{X}^{\eta}$ for deformations of $\bar{\rho}$ of determinant $\eta$ and defined on $\pi_{1}(X)$. In [dJo01] the following is shown.

Theorem 1.3 [dJo01]. Suppose $\bar{\rho}$ is absolutely irreducible when restricted to $\pi_{1}(\bar{X})$. Then Conjecture 1.1 is equivalent to $R_{X}^{\eta}$ being finite (as a module) over $\mathbf{Z}_{\ell}$.

The theorem combined with the result quoted in Remark 1.2(i) shows the following result.

Corollary 1.4 [dJo01]. Suppose $n=2$ and $\bar{\rho}$ is absolutely irreducible when restricted to $\pi_{1}(\bar{X})$. Then $R_{X}^{\eta}$ is finite over $\mathbf{Z}_{\ell}$.

Remark 1.5. Using obstruction theory, de Jong shows [dJo01] that, if $R_{X}^{\eta}$ is finite over $\mathbf{Z}_{\ell}$, then it is also flat over $\mathbf{Z}_{\ell}$ and a complete intersection.

\subsection{Results}

In Theorem 3.1 we shall prove, under a mild restriction, that the $\operatorname{ring} R_{X}^{\eta}$ is finite over $\mathbf{Z}_{\ell}$. To avoid some technicalities, here we only state the following special case.

Theorem 1.6. Let $\bar{\rho}: \pi_{1}(X) \rightarrow \mathrm{SL}_{n}(\mathbf{F})$ be a representation with $\mathbf{F}$ a finite field of characteristic $\ell \neq p$. Assume that $\bar{\rho}$ has full image, $\ell \backslash n,|\mathbf{F}| \geqslant 4$, and $|\mathbf{F}|>5$ if $n=2$. Then the ring $R_{X}^{\eta}$ is finite over $\mathbf{Z}_{\ell}$, and in particular Conjecture 1.1 holds for all $\rho$ with reduction $\bar{\rho}$.

Corollary 1.7. Let $\bar{\rho}$ be as in the previous theorem. Then it lifts to an $\ell$-adic representation $\rho: \pi_{1}(X) \rightarrow \mathrm{SL}_{n}(\mathcal{O})$ with $\mathcal{O}$ the ring of integers of a finite extension of $\mathbf{Q}_{\ell}$. The representation $\rho$ arises from a cuspidal eigenform, and hence $\bar{\rho}$ arises via reduction from a cuspidal eigenform.

If moreover at any place in $S$ ramification is either tame or of order prime to $\ell$, then there exists an $\ell$-adic lift $\rho$ and a corresponding cuspidal eigenform both of the same conductor as $\bar{\rho}$.

Representations $\bar{\rho}$ as above we call automorphic (of minimal conductor).

Proof of Corollary 1.7. We prove the second statement, as the first is similar and easier. Let $R_{X}^{0, \eta}$ denote the quotient of $R_{X}^{\eta}$ which parameterizes deformations which are minimal at the places in $S$. 
(This is a purely Galois-theoretic requirement. The conditions on ramification are needed so that we can formulate, using [BK05, Propositions 5.1 and 5.2], a minimality condition.) By Poitou-Tate and

some obstruction theoretic arguments due to Mazur, it is by now standard to show that $R_{X}^{0, \eta}$ has a presentation $W(\mathbf{F})\left[\left[x_{1}, \ldots, x_{n}\right]\right] /\left(y_{1}, \ldots, y_{n}\right)$, where some of the $y_{i}$ could be zero. Because $R_{X}^{\eta} /(\ell)$ is finite, the same holds for $R_{X}^{0, \eta} /(\ell)$. From this one deduces easily that $R_{X}^{0, \eta}$ must be finite flat over $\mathbf{Z}_{\ell}$. This proves the corollary when combined with the results of [Laf02].

Remark 1.8. The corollary combined with finiteness theorems of Harder about dimensions of cusp forms with bounded conductor and fixed central character has consequences for conjectures in [Kha00], [Moo00] and [MT01]; see [BK06].

\subsection{Sketch of proof}

The form of Conjecture 1.1, combined with Remark 1.2(ii) and Theorem 1.3, lends itself to proving it not necessarily over $X$, but over a suitable finite cover $Y$ of it, i.e. to applying base change techniques. We repeatedly make use of this. In a first reduction, base change allows us to pass to a situation where the assumptions of Theorem 1.6 are satisfied, and moreover $X$ is projective.

In a second reduction step, we apply a level lowering technique of Skinner and Wiles [SW01] to the lift of $\bar{\rho}$ constructed in [BK05]. This relies on an important principle that was discovered by Carayol [Car89] to switch types of automorphic representations that give rise to a given $\bar{\rho}$. The technique of Skinner and Wiles yields, after finite base change, a minimal lift (i.e. a representation of $\pi_{1}(Y)$ with $Y$ projective) of $\bar{\rho}$ over some finite cover $Y$ of $X$. (As the results in [Laf02] provide us with all base change results one expects, this does not require solvable base change.)

Thus it suffices to prove that the deformation ring $R_{Y}^{\eta}$ of a representation $\bar{\rho}: \pi_{1}(Y) \rightarrow \mathrm{SL}_{n}(\mathbf{F})$, with large image in the sense of Theorem 1.6, is finite over $\mathbf{Z}_{\ell}$, where $Y$ is a projective, smooth, geometrically connected curve, and $\bar{\rho}$ lifts to an $\ell$-adic representation of $\pi_{1}(Y)$. (Here $R_{Y}$ is a minimal deformation ring, i.e. parameterizes equivalence classes of lifts of $\bar{\rho}$, to representations of $\pi_{1}(Y)$ with a fixed determinant $\eta$, defined over certain complete Noetherian $\mathbf{Z}_{\ell}$-algebras.) This is a significant simplification, if compared to the situation over number fields, as we need no level raising results (which are still not available, at least in any generality).

We prove the finiteness of $R_{Y}^{\eta}$ by constructing Taylor-Wiles systems for $\bar{\rho}$ using the Galois cohomology techniques of $\S 2$ and automorphic methods of $\S 3$. This allows one to prove that the deformation ring $R_{Y}^{\eta}$ for $\bar{\rho}$ is finite over $\mathbf{Z}_{\ell}$. By what we have said this proves Theorem 1.6.

Our techniques follow closely the original method of Wiles and Taylor in [Wil95] and [TW95], and later developments [Dia97, Fuj, SW01, HT03], which we have to generalize to our context. In fact most of the work of this paper is devoted to carrying out these generalizations. There is a small modification needed to handle problems arising from 'torsion' which may be of relevance even in the number field case: in an Appendix we explain this innovation in the context considered in [Wi195] and [TW95].

\subsection{Some remarks}

In a previous version [BK03] of this paper we had a weaker version of Theorem 1.6 with the additional hypothesis that $\bar{\rho}$ was locally irreducible at some place. A forthcoming result of Ogilvie [Ogi05], see Theorem 3.7 below, allows us to remove this hypothesis.

A proof of all cases of de Jong's conjecture has been announced conditionally by Gaitsgory [Gai04] for $\ell>2$. His methods are different from ours. Our proof works in many cases when $\ell=2$.

There are two separate works of Genestier and Tilouine (for $\mathrm{GSp}_{4}$ ), and Clozel, Harris and Taylor (for $\mathrm{GL}_{n}$ ), on generalizations of [Wi195] and [TW95] to $n$-dimensional representations of absolute Galois groups of totally real number fields. It is a comfort to us that we may quote verbatim 


\section{G. BöCKLe And C. Khare}

from $\S$ II of [HT03] for the Hecke action at places that are introduced in building Taylor-Wiles systems. One of the technical differences between the present work and [HT03] when building Taylor-Wiles systems is that we allow for the possibility that the $\ell$ th roots of 1 are in the base field which entails slight adjustments on the Galois and automorphic side.

The key qualitative difference between the mentioned works and ours is that we can prove automorphy of residual representations like $\bar{\rho}$ in the theorem (by the main result of [BK05]), while in the works quoted this has to be at the moment an important assumption that seems extremely difficult to verify in the number field case. Furthermore our aim is different: we are mainly interested in establishing algebraic properties of deformation rings, while in the number field case these are established en route to proving modularity of $\ell$-adic representations (which is known in our context by [Laf02]).

Throughout this paper we use the notation of Part I of this work without further mention.

\section{Galois cohomology}

\subsection{On deformations of the determinant}

Unlike in Part I of this work, we will no longer work with fixed determinants when considering deformation problems. At the same time, we want to keep finite the number of such determinants. The standard way over function fields to achieve this is to require that under any deformation of the determinant a certain a priori chosen place is totally split. This is basically what we will be doing, except that the deformation theory, for instance in the proof of Lemma 2.9, requires a slight twist of this, cf. Remark 2.10. The choice of place is the content of the following lemma.

Lemma 2.1. There exist infinitely many places $w \in X$ whose residue field $k_{w}$ satisfies $\ell \chi\left[k_{w}: k\right]$ and $k\left(\zeta_{\ell}\right) \cap k_{w}=k$.

Proof. Let $K^{\prime}$ denote the unique constant field extension of $K$ of degree $\ell$. For a place $w$ of $K$ the following three conditions are equivalent: (a) $w$ is split in $K^{\prime} / K$, (b) $\ell$ divides $\left[k_{w}: k\right]$, and (c) $\operatorname{Frob}_{w} \in \operatorname{Gal}\left(K^{\prime} / K\right)$ is trivial. Similarly one has equivalences between $\left(\mathrm{a}^{\prime}\right) k_{w} \cap k\left(\zeta_{\ell}\right)=k$, $\left(\mathrm{b}^{\prime}\right) K\left(\zeta_{\ell}\right) / K$ is inert at $w,\left(\mathrm{c}^{\prime}\right) \operatorname{Frob}_{w} \in \operatorname{Gal}\left(K\left(\zeta_{\ell}\right) / K\right)$ has maximal order. Since $\operatorname{Gal}\left(K\left(\zeta_{\ell}\right) / K\right)$ and $\operatorname{Gal}\left(K^{\prime} / K\right)$ are abelian of relatively prime order, the group $\operatorname{Gal}\left(K^{\prime}\left(\zeta_{\ell}\right) / K\right)$ is the direct product of the above two. Let $\sigma \in \operatorname{Gal}\left(K^{\prime}\left(\zeta_{\ell}\right) / K\right)$ be of maximal order. By the Cebotarev density theorem there exist infinitely many places $w \in X$ whose Frobenius automorphism maps to $\sigma$. Any such $w$ will have the desired properties.

Remark 2.2. The assertions of the Weil conjecture for curves over finite fields allow one to deduce an effective version of the Čebotarev density theorem over function fields. Therefore one may strengthen Lemma 2.1 to the following assertion. There exists an $n_{0} \in \mathbf{N}$ such that for every $n \geqslant n_{0}$ there is a place $w$ of degree $n$ over $k$ which satisfies the conditions of Lemma 2.1.

For a place $w$ as in Lemma 2.1 and $\bar{I}_{w}:=I_{w} /\left(\left[G_{w}, I_{w}\right] I_{w}^{\ell}\right)$ one has a split exact sequence

$$
0 \longrightarrow \bar{I}_{w} \longrightarrow G_{w}^{\mathrm{ab}} \longrightarrow \hat{\mathbf{Z}} \longrightarrow 0,
$$

where the group $\bar{I}_{w} \cong k_{w}^{*} / k_{w}^{* \ell}$ is a quotient of $\mathbf{Z} /(\ell)$ and trivial unless $\zeta_{\ell} \in K_{w}$. We fix a splitting $s_{w}: G_{w}^{\mathrm{ab}} \rightarrow \bar{I}_{w}$.

Lemma 2.3. Let $w$ and $s_{w}$ be as above. Let $\bar{\rho}: G_{w} \rightarrow\{1\} \in \mathbf{F}$ denote the trivial character and $\rho^{w}: G_{w} \rightarrow \mathrm{GL}_{1}\left(R^{w}\right)$ the universal deformation of $\bar{\rho}$ for deformations which factor via the splitting $s_{w}$. Denote by $L_{w, d} \subset H^{1}\left(G_{w}, \mathbf{F}\right)$ the subspace corresponding to the tangent space of the universal deformation. 
Mod $\ell$ REPRESENTATIONS OF ARITHMETIC FUNDAMENTAL GROUPS, II

Then $\operatorname{dim} L_{w, d}=\operatorname{dim}_{\mathbf{F}} H^{0}\left(G_{w}, \mathbf{F}(1)\right)=\operatorname{dim}_{\mathbf{F}} \mathbf{F}(1)^{\pi_{1}(X)}$, the subspace $L_{w, d}$ is a complement to $H_{\mathrm{unr}}^{1}\left(G_{w}, \mathbf{F}\right)$ in $H^{1}\left(G_{w}, \mathbf{F}\right)$, and $L_{w, d}^{\perp}$ is a complement to $H_{\mathrm{unr}}^{1}\left(G_{w}, \mathbf{F}(1)\right)$ in $H^{1}\left(G_{w}, \mathbf{F}(1)\right)$.

Proof. From the definition of $R^{w}$ it follows that $L_{w, d}$ is a complementary sub vector space for $H_{\mathrm{unr}}^{1}\left(G_{w}, \mathbf{F}\right)$ in $H^{1}\left(G_{w}, \mathbf{F}\right)$. Since local Tate-duality is perfect, $L_{w, d}^{\perp}$ must be a complementary vector space for $H_{\mathrm{unr}}^{1}\left(G_{w}, \mathbf{F}(1)\right)$ in $H^{1}\left(G_{w}, \mathbf{F}(1)\right)$. The fact that $L_{w, d}$ is complementary to $H_{\mathrm{unr}}^{1}\left(G_{w}, \mathbf{F}\right)$ also implies that it has the same dimension as $H^{2}\left(G_{w}, \mathbf{F}\right)$. Again by local Tate-duality the latter is equal to the dimension of $H^{0}\left(G_{w}, \mathbf{F}(1)\right)$. Combined with the second assertion in Lemma 2.1 this yields the identities for $\operatorname{dim}_{\mathbf{F}} L_{w, d}$.

From now on, for the remainder of this paper, we fix a place $w \in X$ as in Lemma 2.1 and a splitting $s_{w}$ as in (1).

\subsection{Removing local ramification}

The current section lays the Galois-theoretic groundwork for the base change techniques which we will apply repeatedly in the proof of our main result in the subsequent section. In this respect the following proposition and corollary will be of much use to us.

Proposition 2.4. Let $R$ be in $\mathcal{A}$, let $\rho: \pi_{1}(X) \rightarrow \mathrm{GL}_{n}(R)$ be a continuous representation and let $S_{1} \subset S$ be the set of places at which $\rho\left(I_{v}\right)$ is finite. Let $T_{s}, T_{i} \subset \widetilde{X}$ be finite and disjoint and let $m$ be some positive integer. Then there exists a finite (possibly ramified) Galois cover $Y \rightarrow X$, say with function field $L$, such that:

(i) $L / K$ is totally split at all places above of $T_{s}$;

(ii) at places in $T_{i}$, the residue degree of the extension field $L / K$ is a multiple of $m$;

(iii) the restriction $\rho_{\mid \pi_{1}(Y)}$ is unramified at the places above $S_{1} \backslash T_{s}$.

Note that $S=S_{1}$ if either $R$ is finite or $R$ is the ring of integers of a local field of positive characteristic, cf. Remark 1.2(ii). Before giving the proof, we state the following important corollary, which will be used in the sequel.

Corollary 2.5. Suppose $R$ lies in $\mathcal{A}$ and $\rho, S_{1}$ are as in Proposition 2.4. Let $T_{i}$ be a subset of $\widetilde{X}$ and $m$ some positive integer. Then there exists a finite (possibly ramified) Galois covering $Y \rightarrow X$ with corresponding extension $L / K$ of function fields such that:

(i) $Y$ is geometrically connected over $k$;

(ii) $\rho\left(\pi_{1}(X)\right)=\rho\left(\pi_{1}(Y)\right), \bar{\rho}\left(\pi_{1}(\bar{X})\right)=\bar{\rho}\left(\pi_{1}(\bar{Y})\right)$;

(iii) $\rho_{\mid \pi_{1}(Y)}$ is unramified above the places in $S_{1}$;

(iv) at places above $T_{i}$, the residue degree of $L / K$ is a multiple of $m$.

In particular if $R$ is finite or the ring of integers of a local field of positive characteristic, we can ensure that $\rho_{\mid \pi_{1}(Y)}$ is unramified everywhere.

Proof. We claim that there is a finite set $T^{\prime} \subset X$ disjoint from $T_{i}$ such that the elements $\rho\left(\right.$ Frob $\left._{v}\right)$, $v \in T^{\prime}$, topologically generate $\rho\left(\pi_{1}(X)\right)$. Since $\bar{\rho}\left(\pi_{1}(X)\right)$ is finite, by the Čebotarev density theorem there exists a finite set $T^{\prime \prime} \subset X$ disjoint from $T_{i}$ with the above property for $\bar{\rho}$. Let $X^{\prime} \rightarrow X$ be the smallest finite Galois covering over which $\bar{\rho}$ becomes trivial. Then $\rho\left(\pi_{1}\left(X^{\prime}\right)\right)$ is a pro- $\ell$ group, and since the pro- $\ell$ completion of $\pi_{1}\left(X^{\prime}\right)$ is topologically finitely generated, so is $\rho\left(\pi_{1}\left(X^{\prime}\right)\right)$. Therefore its Frattini quotient is finite, and again by the Cebotarev density theorem we may choose a finite set $T^{\prime \prime \prime} \subset X$ disjoint from $T_{i}$ such that the elements $\rho\left(\right.$ Frob $\left._{v}\right), v \in T^{\prime \prime \prime}$, lie in $\rho\left(\pi_{1}\left(X^{\prime}\right)\right)$ and span the Frattini quotient. Therefore by Burnside's basis theorem these elements topologically generate $\rho\left(\pi_{1}\left(X^{\prime}\right)\right)$. The claim follows with $T^{\prime}:=T^{\prime \prime} \cup T^{\prime \prime \prime}$. 


\section{G. BÖCKLE AND C. Khare}

Recall that $E$ is the splitting field of $\bar{\rho}$. Let now $T_{s} \supset T^{\prime}$ be a finite set of places disjoint from $T_{i}$ such that:

(a) the greatest common divisor of the $q_{v}, v \in T_{s}$, is $q$; and

(b) there exist $w_{1}, \ldots, w_{j} \in T_{s}$ such that Frob $_{w_{i}} \in G_{E}, i=1, \ldots, j$, and such that $\#\left(E \cap \overline{\mathbf{F}}_{p}\right)$ is the greatest common divisor of the $q_{w_{i}}, i=1, \ldots, j$.

Applying Proposition 2.4 to these data, the corollary follows. (Note that condition (b) guarantees the second part of (ii).)

We first prove the following lemma.

Lemma 2.6. Let $\widetilde{K} / K$ be finite with constant field $k$, let $u$ be a place of $\widetilde{K}$ and let $\widetilde{K}_{u}$ be the corresponding completion. Suppose $F / \widetilde{K}_{u}$ is Galois of prime degree $e$. Then for any set of places $T$ of $\widetilde{K}$ not containing $u$, there exists a Galois extension $L$ of $\widetilde{K}$ of degree $e$ such that:

(i) all places in $T$ are split;

(ii) there is a unique place $u^{\prime}$ in $L$ above $u$;

(iii) the extension $L_{u^{\prime}} / \widetilde{K}_{u}$ is isomorphic to $F / \widetilde{K}_{u}$.

Proof. For notational convenience, we give the proof only for $\widetilde{K}=K$. We will construct the desired extension by the use of class field theory. Recall first the left exact sequence

$$
0 \longrightarrow E_{T \cup\{u\}} \longrightarrow K^{*} \longrightarrow \prod_{v \notin T \cup\{u\}} K^{*} / \mathcal{O}_{v}^{*},
$$

where $E_{T \cup\{u\}}$ is the group of $(T \cup\{u\})$-units of $K$. As a group $E_{T \cup\{u\}} \cong k^{*} \times \mathbf{Z}^{\# T}$. Let $f$ be an element of $E_{T \cup\{u\}} \backslash E_{T \cup\{u\}}^{e}$. The extension $K\left[f^{1 / e}\right]$ over $K$ is non-trivial. Hence the number of places at which this extension is not completely split is infinite. By class field theory this means that $f$ lies in $\mathcal{O}_{v}^{*} \backslash \mathcal{O}_{v}^{* e}$ for infinitely many places $v$.

Choose now elements $f_{1}, \ldots, f_{s} \in E_{T \cup\{u\}}$ whose images in $E_{T \cup\{u\}} / E_{T \cup\{u\}}^{e}$ form a basis. By the previous paragraph we may find a finite set of places $\widetilde{T}=\left\{v_{1}, \ldots, v_{s}\right\}$ of $K$ disjoint from $T \cup\{u\}$ and subgroups $U_{v} \subset \mathcal{O}_{v}^{*}$ for $v \in \widetilde{T}$ of index $e$, such that

$$
0 \longrightarrow E_{T \cup\{u\}}^{e} \longrightarrow K^{*} \longrightarrow \prod_{v \notin T \cup\{u\} \cup \widetilde{T}} K^{*} / \mathcal{O}_{v}^{*} \times \prod_{v \in \widetilde{T}} K^{*} / U_{v}
$$

is left exact. From this one easily deduces the injectivity of

$$
K_{u}^{*} / U_{u} \hookrightarrow K^{*} \backslash\left(\prod_{v \notin T \cup\{u\} \cup \widetilde{T}} K_{v}^{*} / \mathcal{O}_{v}^{*} \times \prod_{v \in \widetilde{T}} K_{v}^{*} / U_{v} \times K_{u}^{*} / U_{u}\right),
$$

where $U_{u} \subset K_{u}^{*}$ is the subgroup which via local class field theory corresponds to $F / K_{u}$.

By possibly enlarging $T$, we may assume that the $T$-class group of $K$ is trivial, i.e. that the coordinate ring of $\widetilde{X} \backslash T$ is a unique factorization domain. Then the right-hand side of (2) is $e$-torsion. Since it is finitely generated, as well, it yields an elementary abelian $e$-extension of $K$. Clearly the extension is totally split above $T$. By the injectivity of the map (2) the extension also has the desired decomposition properties at $u$. The existence of an extension of $K$, satisfying (i)-(iii), follows by choosing a suitable summand of the left-hand side of (2).

Proof of Proposition 2.4. By possibly shrinking $S$, we may assume that $\bar{\rho}$ is ramified at all places of $S$. Let $v_{1}, \ldots, v_{r}$ denote the places in $S$ and $v_{1}, \ldots, v_{r^{\prime}}, r^{\prime} \leqslant r$, those in $S_{1} \backslash T_{s}$. The groups $\rho\left(G_{v}\right)$ are all pro-solvable. By repeated application of the above lemma, we first construct a finite extension $L_{1}$ over $K$ which is totally split at all places in $T_{s}$ and such that the local extension of $L_{1} / K$ above $v_{1}$ 
is Galois with group isomorphic to $\rho\left(G_{v_{1}}\right)$ under $\rho$. Then construct $L_{2} / L_{1}$ which is again totally split at all places of $T_{s}$ and such that for a place $v_{2}^{\prime}$ of $L_{1}$ above $v_{2}$ in $K$ the local extension of $L_{2} / L_{1}$ above $v_{1}^{\prime}$ is Galois with group isomorphic to $\rho\left(G_{v_{2}}\right)$ under $\rho$. One reaches inductively an extension $L_{r^{\prime}}$ which contains places $w_{1}, \ldots, w_{r^{\prime}}$ above $v_{1}, \ldots, v_{r^{\prime}}$ such that the restriction of $\rho$ to each $G_{w_{i}}$ is trivial.

By another repeated application of the lemma, we may construct an extension $L_{r^{\prime}}^{\prime}$ of $L_{r^{\prime}}$, totally split at all places above $T_{s}$ and such that the residue degree at places in $T_{i}$ grows by a multiple of $m$. (Locally at places in $T_{i}$ one constructs the unramified extensions of degree $m$.) Then the Galois closure $L$ of $L_{r^{\prime}}^{\prime}$ above $K$ has all the desired properties.

Simplifying ASSUmption. Henceforth, because of the corollary, and Theorem 1.3, we may and will assume that the curve $X$ in Theorem 1.6 is projective. (Note that this simplification already appeared in [dJo01].)

\subsection{On Taylor-Wiles auxiliary primes}

In $\S 3.4$, we will construct Taylor-Wiles systems [TW95] in the minimal case. As such they consist of a Galois-theoretic and a Hecke part. The current section provides the Galois-theoretic tools needed. Recall that in [BK05] the extension $E / K$ is defined as the splitting field $\bar{\rho}$.

We begin with the following lemma.

Lemma 2.7. Let $v$ be a place such that $q_{v} \equiv 1(\bmod \ell), \bar{\rho}$ is unramified at $v$ and $\bar{\rho}\left(\right.$ Frob $\left._{v}\right)$ has distinct eigenvalues which are all contained in $\mathbf{F}$. Let $(R, \mathfrak{M})$ be in $\mathcal{A}$ and let $\rho_{v}: G_{v} \rightarrow \mathrm{GL}_{n}(R)$ be a lift of $\bar{\rho}_{v}$. Then up to strict equivalence the image of $\rho_{v}$ is diagonalizable.

Recall that $\rho_{v}, \rho_{v}^{\prime}: G_{v} \rightarrow \mathrm{GL}_{n}(R)$ are strictly equivalent if there exists $M \in \mathrm{GL}_{n}(R)$ congruent to the identity modulo $\mathfrak{M}$ such that $M \rho_{v} M^{-1}=\rho_{v}^{\prime}$.

Note also that $\rho_{v}$ will factor through the tame quotient $\bar{G}_{q_{v}}$ of $G_{v}$ since $\bar{\rho}$ is unramified and the kernel of $\pi: \mathrm{GL}_{n}(R) \rightarrow \mathrm{GL}_{n}(\mathbf{F})$ is prime to $p$. The lemma implies that in fact $\rho_{v}$ factors through the abelianization $\hat{\mathbf{Z}} \times \mathbf{Z} /\left(q_{v}-1\right)$ of $\bar{G}_{q_{v}}$. The following proof is analogous to the one in Faltings' appendix to [TW95].

Proof. Let us assume that $\bar{\rho}_{v}$ takes its image in the diagonal matrices. This shows, in particular, that the exponent $e$ of the cyclic group $\bar{\rho}\left(G_{v}\right)$ is prime to $\ell$. Because the kernel of $\pi$, defined above, is a pro$\ell$ group, the representation $\rho_{v}: \bar{G}_{q_{v}} \rightarrow \mathrm{GL}_{n}(R)$ must factor via the quotient $G:=\mathbf{Z}_{\ell}(1) \rtimes\left(\mathbf{Z}_{\ell} \times \mathbf{Z} /(e)\right)$ of $\bar{G}_{q_{v}}$.

Let $\sigma$ be a generator of $\mathbf{Z} /(e)$ and $s$ of $\mathbf{Z}_{\ell} \times \mathbf{Z} /(e)$. We may regard $\sigma$ as well as $s$ as elements of $G$. Because $\bar{\rho}(\sigma)$ has distinct eigenvalues and $\rho_{v}(\sigma)$ has finite order $e$, using strict equivalence we may assume that $\rho_{v}(\sigma)$ is diagonal. Since $\bar{\rho}(\sigma)$ has distinct eigenvalues, the same holds for $\rho_{v}(\sigma)$. But this implies that $\rho_{v}(s)$ is diagonal as well, because $\rho_{v}(s)$ commutes with $\rho_{v}(\sigma)$.

Let $t$ be a generator of $\mathbf{Z}_{\ell}(1)$. We claim that $\rho_{v}(t)\left(\bmod \mathfrak{m}^{i}\right)$ is diagonal for all $i \geqslant 1$. Proceeding by induction, the assertion is trivially true for $i=1$. So let us assume that the assertion holds for some $i$ and write $\rho_{v}(t)=D+B$, where $D$ is a diagonal matrix and $B$ is zero along the diagonal and has entries in $\mathfrak{m}^{i}$. Because $B^{2} \equiv 0\left(\bmod \mathfrak{m}^{i+1}\right)$, the relation $s t s^{-1}=t^{q_{v}}$ yields

$$
\rho_{v}(s)(D+B) \rho_{v}(s)^{-1} \equiv D^{q_{v}}+\sum_{i=0}^{q_{v}-1} D^{i} B D^{q_{v}-i-1} \quad\left(\bmod \mathfrak{m}^{i+1}\right) .
$$

As $D \equiv I(\bmod \mathfrak{m})$, and $q_{v}-1 \in \mathfrak{m}$, the right-hand side is congruent to $D^{q_{v}}+B$ modulo $\mathfrak{m}^{i+1}$. Comparing off-diagonal entries, we see that $B\left(\bmod \mathfrak{m}^{i+1}\right)$ commutes with $\rho_{v}(s)\left(\bmod \mathfrak{m}^{i+1}\right)$. This shows that $B\left(\bmod \mathfrak{m}^{i+1}\right)$ is diagonal, and hence zero. 


\section{G. BÖCKLE AND C. Khare}

Let us fix a place $v$ as in the previous lemma, an integer $m \in \mathbf{N}$ such that $\ell^{m} \mid\left(q_{v}-1\right)$, and an eigenvalue $\lambda$ of $\bar{\rho}\left(\operatorname{Frob}_{v}\right)$. The place $v$ is of the type used by Taylor and Wiles [TW95] as an auxiliary prime. By the previous lemma, any deformation of $\bar{\rho}_{\mid G_{v}}$ will have diagonal image. We now define a local deformation problem which further restricts ramification, namely so that it can only occur in the 'direction' defined by the chosen eigenvalue $\lambda$. More precisely, we define the collection of lifts $\mathcal{C}_{v, \lambda, m}$ of $\bar{\rho}_{v}$ as follows.

Let $s, t \in \bar{G}_{q_{v}}^{\mathrm{ab}} \cong \mathbf{Z} /\left(q_{v}-1\right) \times \hat{\mathbf{Z}}$ be such that $t$ generates inertia and $s$ maps to Frob ${ }_{v}$ in $G_{v} / I_{v}$. Denote by $\lambda_{1}, \ldots, \lambda_{n}$ Teichmüller lifts of the eigenvalues if $\bar{\rho}\left(\right.$ Frob $\left._{v}\right)$ such that $\lambda \equiv \lambda_{1}(\bmod \ell)$. Set

$$
R_{v, m}:=W(\mathbf{F})\left[\left[x_{1}, \ldots, x_{n}, y\right]\right] /\left((1+y)^{\ell^{m}}-1\right),
$$

and define $\rho_{v, \lambda, m}: G_{v} \longrightarrow G_{v}^{\mathrm{ab}} \longrightarrow \mathrm{GL}_{n}\left(R_{v, m}\right)$ by

$$
s \mapsto \operatorname{Diag}\left(\lambda_{1}\left(1+x_{1}\right), \ldots, \lambda_{n}\left(1+x_{n}\right)\right), \quad t \mapsto \operatorname{Diag}((1+y), 1,1, \ldots, 1) .
$$

Finally define $\mathcal{C}_{v, \lambda, m}: \mathcal{A} \rightarrow$ Sets by

$$
\begin{aligned}
R \mapsto \mathcal{C}_{v, \lambda, m}(R):=\left\{\rho: G_{v} \rightarrow \operatorname{GL}_{n}(R) \mid \exists \alpha \in \operatorname{Hom}_{\mathcal{A}}\left(R_{v, m}, R\right),\right. \\
\\
\left.M \in 1+M_{n}\left(\mathfrak{m}_{R}\right): \rho=M\left(\alpha \circ \rho_{v, \lambda, m}\right) M^{-1}\right\} .
\end{aligned}
$$

We define $L_{v, \lambda, m} \subset H^{1}\left(G_{v}, \operatorname{ad}(\bar{\rho})\right)$ as the subspace spanned by the 1-cocycles

$$
\left\{c: g \mapsto \frac{1}{\varepsilon}\left(\rho(g) \rho_{0}^{-1}(g)-I\right) \mid \rho \in \mathcal{C}_{v, \lambda, m}\left(\mathbf{F}[\varepsilon] /\left(\varepsilon^{2}\right)\right)\right\},
$$

where $\rho_{0}$ is the tautological lift induced from the splitting $\mathbf{F} \rightarrow \mathbf{F}[\varepsilon] /\left(\varepsilon^{2}\right)$. It is easy to see that $\operatorname{dim} L_{v, \lambda, m}=\operatorname{dim} H_{\mathrm{unr}}^{1}\left(G_{v}, \operatorname{ad}(\bar{\rho})\right)+1=n+1$. Moreover $L_{v, \lambda, m}$ is independent of $m$ as long as $m>0$. Finally, note also that Corollary 4.9 of [BK05] yields the following lemma.

Lemma 2.8. Let $\sigma \in \operatorname{Gal}\left(E\left(\zeta_{\ell}\right) / K\right)$ be the image of $\operatorname{Frob}_{v}$. Then the subspace

$$
L_{v, \lambda, m}^{\perp} \subset H_{\mathrm{unr}}^{1}\left(G_{v}, \operatorname{ad}(\bar{\rho})(1)\right) \cong \operatorname{ad}(\bar{\rho}) /\left(\operatorname{Frob}_{v}-1\right) \operatorname{ad}(\bar{\rho})
$$

of codimension one only depends on $\sigma$ and the choice of $\lambda$ (among the eigenvalues of $\bar{\rho}(\sigma)$ ).

Because of the above lemma, we also write $L_{\sigma, \lambda}^{\perp}$ or $L_{v, \lambda}^{\perp}$ for $L_{v, \lambda, m}^{\perp}$. Note that since the cyclotomic character $\chi$ is trivial on $G_{v}$, the restrictions of $\operatorname{ad}(\bar{\rho})$ and $\operatorname{ad}(\bar{\rho})(1)$ to $G_{v}$ agree.

The central result of this section is the following which is modeled on [HT03, Theorem IV.5.3]. The notation for Selmer groups is a standard one and for instance the one used in [BK05], and introduced there on page 9 .

Lemma 2.9. Let $\bar{\rho}: \pi_{1}(X) \rightarrow \mathrm{GL}_{n}(\mathbf{F})$ be a continuous representation. Define $L_{w}:=H_{\text {unr }}^{1}\left(G_{w}, \operatorname{ad}^{0}(\bar{\rho})\right)$ $\oplus L_{w, d}$, where $L_{w, d}$ is the subspace of $H^{1}\left(G_{w}, \mathbf{F}\right)$ considered in Lemma 2.3, and $\mathbf{F} \subset \operatorname{ad}(\bar{\rho})$ via the diagonal embedding. Suppose that the following hold.

(i) For any $\pi_{1}(X)$-subrepresentation $V$ of $\operatorname{ad}(\bar{\rho})$, there exists a regular semisimple $g_{V} \in \bar{\rho}\left(\pi_{1}(\bar{X})\right)$ such that $V^{g_{V}} \neq 0$.

(ii) If $\zeta_{\ell} \in E$, then $H^{1}\left(\operatorname{Gal}\left(E / K\left(\zeta_{\ell}\right)\right), \operatorname{ad}^{0}(\bar{\rho})\right)=0$.

(iii) If $\zeta_{\ell} \in K$, then $\operatorname{ad}^{0}(\bar{\rho})$ has no one-dimensional subrepresentation.

(iv) The image of $\bar{\rho}$ has no quotient of order $\ell$.

Then for any given $m \in \mathbf{N}$ there exists a set $Q_{m}$ of $\operatorname{dim} H_{\left\{L_{w}\right\}}^{1}(\{w\}, \operatorname{ad}(\bar{\rho}))$ places of $X \backslash\{w\}$ and there exist elements $\lambda_{v} \in \mathbf{F}$ for all $v \in Q_{m}$ such that:

(a) for all $v \in Q_{m}$ one has the congruence $q_{v} \equiv 1\left(\bmod \ell^{m}\right)$;

(b) for all $v \in Q_{m}$ the matrix $\bar{\rho}\left(\right.$ Frob $\left._{v}\right)$ has $n$ distinct eigenvalues and $\lambda_{v}$ is among them; 
(c) $H_{\left\{L_{v}\right)}^{1}\left(\{w\} \cup Q_{m}, \operatorname{ad}(\bar{\rho})(1)\right)=0$ if one sets $L_{v}:=L_{v, \lambda_{v}}$ for each $v \in Q_{m}$;

(d) $\operatorname{dim} H_{\left\{L_{w}\right\}}^{1}(\{w\}, \operatorname{ad}(\bar{\rho}))=\operatorname{dim} H_{\left\{L_{v}\right\}}^{1}\left(Q_{m} \cup\{w\}, \operatorname{ad}(\bar{\rho})\right)$.

The proof in fact shows that the sets $Q_{m}$ above may be chosen disjoint from any given finite set of places $S^{\prime}$. Moreover the proof can be extended, almost verbatim, to the case where one is given a set of deformation conditions $\left(\mathcal{C}_{v}, L_{v}\right)_{v \in S \cup\{w\}}$ at a finite set $S$ disjoint from $\{w\}$.

Proof. The proof follows closely the analogous proof given in [HT03] which in turn is similar to that in [TW95]. First, one has $h_{\mathrm{unr}}^{1}\left(G_{v}, \operatorname{ad}(\bar{\rho})\right)=h^{0}\left(G_{v}, \operatorname{ad}(\bar{\rho})\right)$ for any place of $X$. Also $\operatorname{dim} L_{w, d}=$ $\operatorname{dim} H^{0}\left(G_{w}, \mathbf{F}(1)\right)$ by Lemma 2.3. Hence [BK05, Remark 3.5], yields

$$
\operatorname{dim} H_{\left\{L_{w}\right\}}^{1}(\{w\}, \operatorname{ad}(\bar{\rho}))=\operatorname{dim} H_{\left\{L_{w}\right\}}^{1}(\{w\}, \operatorname{ad}(\bar{\rho})(1)) .
$$

Define $E_{m}:=E\left(\zeta_{\ell^{m}}\right)$ and let $Y_{m} \rightarrow X \backslash\{w\}$ be the corresponding Galois cover. We first claim that the composite

$$
\begin{aligned}
H_{\left\{L_{w}^{\perp}\right\}}^{1}(\{w\}, \operatorname{ad}(\bar{\rho})(1)) & \hookrightarrow H^{1}\left(\pi_{1}(X \backslash\{w\}), \operatorname{ad}(\bar{\rho})(1)\right) \\
& \rightarrow H^{1}\left(\pi_{1}\left(Y_{m}\right), \operatorname{ad}(\bar{\rho})(1)\right)^{\operatorname{Gal}\left(E_{m} / K\right)}
\end{aligned}
$$

is injective, where the second morphism is restriction.

We have $L_{w}=H_{\mathrm{unr}}^{1}\left(G_{w}, \operatorname{ad}^{0}(\bar{\rho})\right) \oplus L_{w, d}$ with $L_{w, d} \subset H^{1}\left(G_{w}, \mathbf{F}\right)$, and so we may prove the claim separately for the subrepresentations $\mathbf{F}$ and $\operatorname{ad}^{0}(\bar{\rho})$ of $\operatorname{ad}(\bar{\rho})$. We first consider $\operatorname{ad}^{0}(\bar{\rho})$.

Condition (ii) yields $H^{1}\left(\operatorname{Gal}\left(E_{1} / K\right), \operatorname{ad}^{0}(\bar{\rho})(1)\right)=0$, as can be seen by applying for instance [Böc03, Proposition 1.8(i),(ii)]. This proves the claim for $m=1$ and $\operatorname{ad}^{0}(\bar{\rho})$. For $m>1$, inflationrestriction and taking invariants yields the left exact sequence

$$
\begin{aligned}
0 & \rightarrow H^{1}\left(\operatorname{Gal}\left(E_{m} / E_{1}\right), \operatorname{ad}^{0}(\bar{\rho})(1)\right)^{\operatorname{Gal}\left(E_{1} / K\right)} \\
& \rightarrow H^{1}\left(\pi_{1}\left(Y_{1}\right), \operatorname{ad}^{0}(\bar{\rho})(1)\right)^{\operatorname{Gal}\left(E_{1} / K\right)} \\
& \rightarrow H^{1}\left(\pi_{1}\left(Y_{m}\right), \operatorname{ad}^{0}(\bar{\rho})(1)\right)^{\operatorname{Gal}\left(E_{m} / K\right)} .
\end{aligned}
$$

We will show that the left-hand term vanishes. Since $K\left(\zeta_{\ell^{m}}\right)$ is Galois over $K$, the group $\operatorname{Gal}\left(E_{m} / E_{1}\right)$ lies in the center of $\operatorname{Gal}\left(E_{m} / K\right)$. Moreover by the definition of $E_{1}$, the action of $\operatorname{Gal}\left(E_{m} / E_{1}\right)$ on $\operatorname{ad}(\bar{\rho})(1)$ is trivial. Therefore we find

$$
\begin{aligned}
& H^{1}\left(\operatorname{Gal}\left(E_{m} / E_{1}\right), \operatorname{ad}^{0}(\bar{\rho})(1)\right)^{\operatorname{Gal}\left(E_{1} / K\right)} \\
& \quad=H^{1}\left(\operatorname{Gal}\left(E_{m} / E_{1}\right), \mathbf{F}\right) \otimes_{\mathbf{F}}\left(\operatorname{ad}^{0}(\bar{\rho})(1)\right)^{\operatorname{Gal}\left(E_{1} / K\right) .}
\end{aligned}
$$

By (iii) the last expression is zero. This proves the claim for the $\operatorname{ad}^{0}(\bar{\rho})$-component.

We will now consider the diagonal F-component. By inflation-restriction we need to show that

$$
0=H_{\left\{L_{v, d}^{\perp}\right.}^{1}(\{w\}, \mathbf{F}(1)) \cap H^{1}\left(\operatorname{Gal}\left(E_{m} / K\right), \mathbf{F}(1)\right)
$$

inside $H^{1}\left(\pi_{1}(X \backslash\{w\}), \mathbf{F}(1)\right)$. Again by inflation-restriction, the second term allows the isomorphism

$$
H^{1}\left(\operatorname{Gal}\left(E_{m} / K\right), \mathbf{F}(1)\right) \cong\left(H^{1}\left(\operatorname{Gal}\left(E_{m} / K\left(\zeta_{\ell}\right)\right), \mathbf{F}\right) \otimes \mathbf{F}(1)\right)^{\operatorname{Gal}\left(K\left(\zeta_{\ell}\right) / K\right)} .
$$

By assumption (iv) the right-hand side is isomorphic to

$$
H^{1}\left(\operatorname{Gal}\left(K\left(\zeta_{\ell^{m}}\right) / K\left(\zeta_{\ell}\right)\right), \mathbf{F}\right) \otimes\left(\mathbf{F}(1)^{\operatorname{Gal}\left(K\left(\zeta_{\ell}\right) / K\right)}\right) .
$$

If $\zeta_{\ell} \notin K$, the proof of the claim is thus complete. In the case $\zeta_{\ell} \in K$, a non-zero class in $H^{1}\left(\operatorname{Gal}\left(E_{m} / K\right), \mathbf{F}(1)\right)$ describes a non-zero character $\operatorname{Gal}\left(K\left(\zeta_{\ell^{m}}\right) / K\left(\zeta_{\ell}\right)\right) \longrightarrow \mathbf{F}$. Because $\ell \chi\left[k_{w}: k\right]$, such a class restricts to a non-zero class in $H_{\mathrm{unr}}^{1}\left(G_{w}, \mathbf{F}(1)\right)$. By Lemma 2.3 it maps to a non-zero 


\section{G. BÖCKLE AND C. Khare}

class in $H^{1}\left(G_{w}, \mathbf{F}(1)\right) / L_{w, d}^{\perp}$ and so such a class cannot lie in $H_{\left\{L_{w, d}^{\perp}\right\}}^{1}(\{w\}, \mathbf{F}(1))$. This completes the proof of the claim.

Let now $\psi$ be a 1-cocycle whose class $[\psi] \in H_{\left\{L_{w}^{\perp}\right\}}^{1}(\{w\}, \operatorname{ad}(\bar{\rho})(1))$ is non-zero. By the above claim, the restriction of $\psi$ to $\pi_{1}\left(Y_{m}\right)$ is non-trivial. Since $\pi_{1}\left(Y_{m}\right)$ acts trivially on $\operatorname{ad}(\bar{\rho})(1)$, the class $[\psi]$ induces a non-trivial $\operatorname{Gal}\left(E_{m} / K\right)$-equivariant homomorphism $\pi_{1}\left(Y_{m}\right) \rightarrow \operatorname{ad}(\bar{\rho})(1)$. Let $E_{\psi}$ denote the fixed field of its kernel, and $V(1)$ its image in $\operatorname{ad}(\bar{\rho})(1)$. Then the induced morphism $\operatorname{Gal}\left(E_{\psi} / E_{m}\right) \rightarrow V(1)$ is bijective.

Choose a regular semisimple $g \in \bar{\rho}(\bar{X})$ such that $V^{g} \neq 0$, and an element $\sigma \in \operatorname{Gal}\left(E_{1} / K\left(\zeta_{\ell}\right)\right)$ such that $g=\bar{\rho}(\sigma)$. For such a $g$ we now consider

$$
V(1)_{g} \cong V_{g} \longleftrightarrow \operatorname{ad}(\bar{\rho})(1)_{g} \cong \operatorname{ad}(\bar{\rho})_{g} \cong H_{\text {unr }}^{1}\left(G_{v}, \operatorname{ad}(\bar{\rho})(1)\right),
$$

where $v \in X \backslash\{w\}$ is any place with Frob $_{v} \mapsto \sigma$. For an eigenvalue $\lambda$ of $g$ we denote by $L_{g, \lambda}^{\perp} \subset$ $\operatorname{ad}(\bar{\rho})(1)_{g}$ the corresponding subspace as defined in (4). One easily shows $\bigcap_{\lambda} L_{g, \lambda}^{\perp}=0$, where the intersection ranges over all eigenvalues of $g$, e.g. by proving the dual assertion. We claim that there exists $x_{V} \in V_{g}$ and an eigenvalue $\lambda$ of $g$ such that

$$
\psi\left(\operatorname{Frob}_{v}\right)+x_{V} \notin L_{g, \lambda}^{\perp} \subset H_{\mathrm{unr}}^{1}\left(G_{v}, \operatorname{ad}(\bar{\rho})(1)\right) .
$$

Assume otherwise. Then $\psi\left(\operatorname{Frob}_{v}\right)+V_{g} \subset L_{g, \lambda}^{\perp}$ for all eigenvalues $\lambda$. This implies $V_{g} \subset L_{g, \lambda}^{\perp}$ for all $\lambda$, and therefore $V_{g} \subset \bigcap_{\lambda} L_{g, \lambda}^{\perp}=0$, contradicting $V_{g} \cong V^{g} \neq 0$.

Let $\tau \in \pi_{1}(X \backslash\{w\})$ by any element which acts trivially on $E_{m}$ and maps to $x_{V}$ under $\psi$. Because $E_{\psi} / K$ is Galois, by the Cebotarev density theorem there exists a place $v^{\prime} \in X \backslash\{w\}$ such that the image of $\operatorname{Frob}_{v^{\prime}}$ in $\operatorname{Gal}\left(E_{\psi} / K\right)$ agrees with that of $\tau \operatorname{Frob}_{v}$. By the above $[\psi] \notin L_{v^{\prime}, \lambda}^{\perp}$. A simple inductive argument now finishes the proof of the lemma.

Remark 2.10. At two instances the above proof makes crucial use of the special choice of $w$ and of the deformation condition on the determinant that we enforce at $w$. First this choice is needed to obtain formula (5). Second, it is needed to prove the injectivity on the $\mathbf{F}$-component of $\operatorname{ad}(\bar{\rho})$ in the homomorphism displayed below (5).

In the function field situation the extension $K\left(\zeta_{\ell^{m}}\right) / K\left(\zeta_{\ell}\right)$ is a constant field extension, and hence unramified. The special choice of determinantal deformations at $w$ is needed to rule out certain unramified characters. In the number field case the extension $K\left(\zeta_{\ell^{m}}\right) / K\left(\zeta_{\ell}\right)$ typically is ramified at the prime $\ell$, and so a choice of $w$ and a deformation condition is unnecessary.

To complement Lemma 2.9, in the particular case in which we are interested, we also prove the following lemma.

Lemma 2.11. Suppose $\bar{\rho}: \pi_{1}(X) \rightarrow \mathrm{SL}_{n}(\mathbf{F})$ is surjective, $\ell \nmid n,|\mathbf{F}| \geqslant 4$, and $|\mathbf{F}|>5$ for $n=2$. Then:

(i) $\bar{\rho}\left(\pi_{1}(\bar{X})\right)=\mathrm{SL}_{n}(\mathbf{F})$ contains a regular semisimple element;

(ii) $\operatorname{ad}(\bar{\rho})=\operatorname{ad}^{0}(\bar{\rho}) \oplus \mathbf{F}, \operatorname{ad}^{0}(\bar{\rho})$ is irreducible, and for any regular semisimple $g \in \mathrm{SL}_{n}(\mathbf{F})$ one has $\mathbf{F}^{g}=\mathbf{F}$ and $M_{n}^{0}(\mathbf{F})^{g} \neq 0$

(iii) $H^{1}\left(\mathrm{SL}_{n}(\mathbf{F}), M_{n}^{0}(\mathbf{F})\right)=0$;

(iv) $\mathrm{SL}_{n}(\mathbf{F})$ has no quotient isomorphic to $\mathbf{Z} /(\ell)$.

Proof. Under our conditions on $n$ and $\mathbf{F}$, the group $\mathrm{SL}_{n}(\mathbf{F})$ has no abelian quotients, which shows (iv) and $\bar{\rho}(\bar{X})=\mathrm{SL}_{n}(\mathbf{F})$. Parts (i) and (ii) are clear and (iii) follows from [CPS75]. 


\section{Automorphic methods}

We begin this chapter by briefly reviewing parts of the theory of automorphic forms over function fields. We formulate in $\S 3.3$ a principle of Carayol [Car89] in a form suitable for us (cf. the two lemmas in the section). Section 3.4 explains how to carry over the method of Taylor-Wiles systems and its later simplification, cf. [TW95, Dia97], from the number field to the function field case, using crucial results of $\S 3.3$, assuming that we are in a situation where we have an automorphic lift of $\bar{\rho}$ of minimal level. Thereby we prove isomorphisms between certain universal deformation rings and corresponding Hecke algebras, under this crucial assumption. We present a slight technical improvement over the usual method that might also be useful in the number field case. In $\S 3.5$, we prove a theorem on 'lowering the level up to base change' for certain cuspidal Hecke eigenforms, which allows us to verify this assumption in a significant number of cases, enough to cover the applications to Theorems 3.1 and 1.6 that are proved in the last section by pulling all the results of this section together. Our method here is that of Skinner and Wiles [SW01], and again relies on results of $\S 3.3$.

We keep the hypothesis that $X$ is projective and fix some notation to state the main theorem of the final section. Let $\mathcal{O}$ be a discrete valuation ring finite over $W(\mathbf{F})$ and with maximal ideal $\mathfrak{m}$. For a finite subset $T$ of $X$, consider a representation $\rho: \pi_{1}(X \backslash T) \rightarrow \mathrm{GL}_{n}(\mathcal{O})$ with residual representation $\bar{\rho}:=\rho(\bmod \mathfrak{m})$. The extension $E / K$ is as in $\S 2.3$. We say that $\rho$ is type-1 at a place $v \in X$, if $\bar{\rho}$ is unramified at $v$ and $\rho\left(I_{v}\right)$ is unipotent of rank 1 .

In the final section, we prove the following theorem and give some further applications of it.

Theorem 3.1. Let $\mathcal{O}, T, \rho$ and $\bar{\rho}$ be as above and suppose that they satisfy the following.

(i) The representation $\rho$ is type- 1 at all $v \in T$.

(ii) For any $\pi_{1}(X)$-subrepresentation $V$ of $\operatorname{ad}(\bar{\rho})$, there exists a regular semisimple $g_{V} \in \bar{\rho}\left(\pi_{1}(\bar{X})\right)$ such that $V^{g_{V}} \neq 0$.

(iii) If $\zeta_{\ell} \in K$, then $\operatorname{ad}^{0}(\bar{\rho})$ has no one-dimensional subrepresentation.

(iv) If $\zeta_{\ell} \in E$, then $H^{1}\left(\operatorname{Gal}\left(E / K\left(\zeta_{\ell}\right)\right)\right.$, $\left.\operatorname{ad}^{0}(\bar{\rho})\right)=0$.

(v) The image of $\bar{\rho}$ has no quotient of order $\ell$.

(vi) Finally, $\eta:=\operatorname{det} \rho$ is of finite order.

Then $R_{X}^{\eta}$ is finite flat over $\mathbf{Z}_{\ell}$.

The proof of this theorem will occupy us in the rest of this paper. Combined with the Galoistheoretic lifting results of [BK05, Theorems 1.1 and 2.4], the above theorem will easily imply Theorem 1.6 as we see at the very end (see proof of Theorem 4.1).

Note that if $\operatorname{ad}^{0}(\bar{\rho})$ is (absolutely) irreducible, then so is $\bar{\rho}$. So when we require both assumptions, we only state the former.

The above result would also go through if one allows unipotent ramification of any rank at the places in $T$. However, since we do not need this and since it would make the proofs unnecessarily technical, we content ourselves with the rank one case.

Let us fix the following notation for this section. For a place $v$ of $\widetilde{X}$ we denote by $K_{v}, A_{v}, \mathfrak{M}_{v}$, $k_{v}$ and $q_{v}$ the completion of the function field $K$ of $X$ at $v$, its ring of integers, the maximal ideal of the latter, its residue field and the cardinality of the latter, respectively. Let $\mathbf{A}=\mathbf{A}_{K}$ be the adeles over $K$. The symbols $F, \mathcal{O}, \mathbf{F}$ and $\mathfrak{m}$ will denote a sufficiently large finite extension of $\mathbf{Q}_{\ell}$, its ring of integers, its residue field and the maximal ideal of $\mathcal{O}$, respectively. In the definitions to come, $\Lambda$ stands for any of the three rings $F, \mathcal{O}$ or $\mathbf{F}$. 


\section{G. BÖCKLE AND C. Khare}

Note again that we have chosen a place $w \in X$ and a splitting $s_{w}$ as in Lemma 2.1 and in the short exact sequence (1). To this splitting corresponds the choice of a uniformizer $\pi_{w}$ of $K_{w}$ unique up to multiplication by $1+\mathfrak{M}_{w}$. From now on $v$ will always be a place of $K$ different from $w$.

Let $\bar{U}_{0}\left(k_{v}\right) \subset \operatorname{GL}_{n}\left(k_{v}\right)$ be the maximal parabolic which fixes the subspace $e_{1} k_{v}$ and define $U_{0}(v):=\left\{g \in \mathrm{GL}_{n}\left(A_{v}\right): g\left(\bmod \mathfrak{M}_{v}\right) \in \bar{U}_{0}(v)\right\}$. For $\bar{g} \in \bar{U}_{0}\left(k_{v}\right)$ denote by $a_{11}(\bar{g})$ its $(1,1)$-entry.

Definition 3.2. For a character $\chi_{v}: k_{v}^{*} \rightarrow \Lambda^{*}$, we denote by $I_{1}\left(\chi_{v}\right)$ the one-dimensional representation of $U_{0}(v)$ defined by

$$
U_{0}(v) \stackrel{\left(\bmod \mathfrak{M}_{v}\right)}{\longrightarrow} \bar{U}_{0}\left(k_{v}\right) \stackrel{a_{11}}{\longrightarrow} k_{v}^{*} \stackrel{\chi_{v}}{\longrightarrow} \Lambda^{*}
$$

We define $U_{1, m}(v):=\left\{g \in U_{0}(v): a_{11}(g(\bmod \mathfrak{m})) \in k_{v}^{* \ell^{m}}\right\}$, so that $U_{1, m}(v) \subset \operatorname{ker}\left(I_{1}\left(\chi_{v}\right)\right) \subset$ $U_{0}(v)$ for any character $\chi_{v}$ of order dividing $\ell^{m}$.

We also define the compact open group $U_{d}(w)$ as the kernel of the composite

$$
\mathrm{GL}_{n}\left(A_{w}\right) \stackrel{\bmod \mathfrak{M}_{w}}{\longrightarrow} \mathrm{GL}_{n}\left(k_{w}\right) \stackrel{\operatorname{det}}{\longrightarrow} k_{w}^{*} \longrightarrow k_{w}^{*} /\left(k_{w}^{* \ell}\right) .
$$

\subsection{Automorphic forms over function fields}

Our next aim is to define spaces of cusp forms on $\mathrm{GL}_{n}(\mathbf{A})$.

In the sequel we will often work under the following hypothesis.

\section{Assumption 3.3.}

(i) $T$ is a finite subset of $X \backslash\{w\}$.

(ii) $\omega: \mathrm{GL}_{1}(\mathbf{A}) \rightarrow \Lambda^{*}$ is a character of finite order, unramified outside $w$, and trivial on $\pi_{w}^{\mathbf{Z}}\left(1+\mathfrak{M}_{w}\right) k_{w}^{* \ell} K^{*}$ (with $\pi_{w} \in K_{w}$ the uniformizer at $w$ chosen above corresponding to the splitting $\left.s_{w}\right)$.

(iii) For $v \in T, \chi_{v}: k_{v}^{*} \rightarrow \Lambda^{*}$ is a character of $\ell$-power order $\ell^{m_{v}}$, which may be trivial.

The above set-up will be of use in two different instances, namely in lowering the level of an automorphic cusp form associated to a residual Galois representation and in constructing TaylorWiles systems. In the first case, we will use the above notation as stated. In the second, the set $T$ will be denoted by $Q$ or $Q_{m}$.

For any $m \in \mathbf{N}_{0}$ we define

$$
Z_{T}^{m}:=K^{*}\left(\pi_{w}^{\mathbf{Z}}\left(1+\mathfrak{M}_{w}\right) k_{w}^{* \ell} \times \prod_{v \in T}\left(1+\mathfrak{M}_{v}\right) k_{v}^{* \ell^{m}} \times \prod_{v \notin T \cup\{w\}} A_{v}^{*}\right) .
$$

For any $m \in \mathbf{N}$ and any finite (possibly empty) $T \subset X \backslash\{w\}$ we define a compact open subgroup of $\mathrm{GL}_{n}(\mathbf{A})$ by

$$
U_{T}^{m}:=U_{d}(w) \times \prod_{v \in T} U_{1, m}(v) \times \prod_{v \notin T \cup\{w\}} \operatorname{GL}_{n}\left(A_{v}\right) \subset \mathrm{GL}_{n}(\mathbf{A}) .
$$

Under the hypothesis of Assumption 3.3, and for $m \geqslant \max \left\{m_{v}: v \in T\right\}$, we define $\mathbf{C}_{\text {cusp,T }}^{\omega,\left(\chi_{v}\right)}(\Lambda)$ as the space of all functions

$$
f: \mathrm{GL}_{n}(K) \backslash \mathrm{GL}_{n}(\mathbf{A}) / U_{T}^{m} \rightarrow \Lambda
$$

with the following properties:

(i) the central action of $Z_{T}^{m}$ on $f$ is described by $\omega$;

(ii) for $v \in T$ the right action of $U_{0}(v)$ on $f\left(g \cdot{ }_{-}\right)$is via the character $I_{1}\left(\chi_{v}\right)$;

(iii) $f$ is cuspidal (cf. $[$ BJ79, $\S 5]$ ). 
Note that the conditions do completely determine the central action of $A_{v}^{*}$. We define the analogous space $\mathbf{C}_{c, T}^{\omega,\left(\chi_{v}\right)}(\Lambda)$ with the last condition replaced by compact support mod center. By a result of Harder we have an inclusion $\mathbf{C}_{\text {cusp }, T}^{\omega,\left(\chi_{v}\right)}(\Lambda) \subset \mathbf{C}_{c, T}^{\omega,\left(\chi_{v}\right)}(\Lambda)$.

For any $m \in \mathbf{N}_{0}$ we define a second space of functions $\mathbf{C}_{\text {cusp,T }}^{\omega, m}(\Lambda)$ as the space of all functions

$$
f: \mathrm{GL}_{n}(K) \backslash \mathrm{GL}_{n}(\mathbf{A}) / U_{T}^{m} \rightarrow \Lambda
$$

such that for $z \in Z_{T}^{m}$ one has $f(z g)=\omega(z) f(g)$, and $f$ is cuspidal. If instead of cuspidal we consider functions that are compact mod center, we denote the corresponding space by $\mathbf{C}_{c, T}^{\omega, m}(\Lambda)$.

The following is an immediate consequence of the above definitions.

Proposition 3.4. Assume that $F$ contains $\zeta_{\ell^{m}}$. Then

$$
\mathbf{C}_{\mathrm{cusp}, T}^{\omega, m}(F) \cong \bigoplus_{\left(\chi_{v}^{\prime}\right)} \mathbf{C}_{\mathrm{cusp}, T}^{\omega,\left(\chi_{v}^{\prime}\right)}(F)
$$

and

$$
\mathbf{C}_{c, T}^{\omega, m}(F) \cong \bigoplus_{\left(\chi_{v}^{\prime}\right)} \mathbf{C}_{c, T}^{\omega,\left(\chi_{v}^{\prime}\right)}(F),
$$

where the $\left(\chi_{v}^{\prime}\right)$ range over all characters of $\prod_{v \in T} U_{0}(v) / U_{1, m}(v)$.

Proposition 3.5. Let $M(\mathcal{O})$ be any of the spaces $\mathbf{C}_{\text {cusp }, T}^{\omega,\left(\chi_{v}\right)}(\mathcal{O}), \mathbf{C}_{c, T}^{\omega,\left(\chi_{v}\right)}(\mathcal{O}), \mathbf{C}_{\text {cusp }, T}^{\omega, m}(\mathcal{O})$ or $\mathbf{C}_{c, T}^{\omega, m}(\mathcal{O})$.

(i) The spaces $M(\mathcal{O})$ are free and when we consider the cuspidal space they are also finitely generated over $\mathcal{O}$.

(ii) The induced morphism $M(\mathcal{O}) \otimes_{\mathcal{O}} \mathbf{F} \rightarrow M(\mathbf{F})$ is injective.

Proof. The modules $\mathbf{C}_{c, T}^{\omega,\left(\chi_{v}\right)}(\mathcal{O})$ and $\mathbf{C}_{c, T}^{\omega, m}(\mathcal{O})$ may be viewed as spaces of finitely supported functions on an infinite (discrete) set. Hence they are free $\mathcal{O}$-modules. The other two are submodules of these, and thus they are free as well. This proves (i).

For (ii) note that the argument given in (i) shows that for the spaces describing compactly supported functions the morphism in question is an isomorphism. Since cuspidality is preserved under the reduction map $\mathcal{O} \rightarrow \mathbf{F}$ the injectivity also follows in the remaining cases.

By definition, cf. [BJ79], one has a smooth admissible automorphic representation $\Pi(f)$ of $\mathrm{GL}_{n}(\mathbf{A})$ attached to an automorphic form $f$ for $\mathrm{GL}_{n}$ (simply given by $F\left[\mathrm{GL}_{n}(\mathbf{A})\right] f$ ). The local constituents of $\Pi(f)$ are denoted $\Pi_{v}(f)$, so that $\Pi(f) \cong \hat{\bigotimes}_{v} \Pi_{v}(f)$. Conversely, if $\Pi$ is a smooth admissible cuspidal automorphic representation and $U$ a compact open subgroup of $\mathrm{GL}_{n}(\mathbf{A})$, then $\Pi^{U}$ is a (possibly empty) space of cusp forms.

\subsection{Hecke algebras}

For $v \notin T \cup\{w\}$ we define the spherical Hecke algebra $\mathcal{H}_{v}$ as the algebra of bi-GL $\mathrm{G}_{n}\left(A_{v}\right)$-invariant locally constant compactly supported functions on $\mathrm{GL}_{n}\left(K_{v}\right)$ with values in $\mathbf{Z}_{\ell}$, and where multiplication is given by convolution. The algebra $\mathcal{H}_{v}$ contains naturally defined elements $T_{v, i}, i=1, \ldots, n$, the Hecke operators at $v$, and the Satake isomorphism asserts that

$$
\mathcal{H}_{v} \cong \mathbf{Z}_{\ell}\left[T_{v, 1}, \ldots, T_{v, n}, T_{v, n}^{-1}\right] .
$$

For $v \in T$ ( $T$ might be empty) and $m_{v} \in \mathbf{N}_{0}$, we follow [HT03, ch. II], (there is no difference between the function and the number field case here). So we denote by $V_{v, i}, i=1, \ldots, n-1$, the Hecke operators in the convolution algebra of $U_{1, m_{v}}(v)$-bi-invariant locally constant compactly supported functions on $\mathrm{GL}_{n}\left(K_{v}\right)$ with values in $\mathbf{Z}_{\ell}$, defined as in [HT03, II.2.2]. By $V_{v, n}$ we denote 


\section{G. BÖCKLE AND C. Khare}

the $U$-operator from [HT03, II.2.4], which again lies in the above convolution algebra. (We choose the notation $V_{v, n}$, to avoid any conflict with our notation for compact opens.) The commutative subalgebra generated over $\mathbf{Z}_{\ell}$ by the $V_{v, i}, i=1, \ldots, n$, is denoted by $\mathcal{H}_{v}^{m_{v}}$. We consider the Hecke action at places in $T$ only when building Taylor-Wiles systems.

To obtain a Hecke action at almost all places (namely outside $w$ ), we set

$$
\mathcal{H}_{T}^{a b, m}:=\bigotimes_{v \in T} \mathcal{H}_{v}^{m} \otimes \bigotimes_{v \notin\{w\} \cup T} \mathcal{H}_{v}
$$

This Hecke algebra acts on the spaces of cusp forms $\mathbf{C}_{\text {cusp }, T}^{\omega,\left(\chi_{v}\right)}(\Lambda), \mathbf{C}_{\mathrm{cusp}, T}^{\omega, m}(\Lambda)$ and the analogous (much larger, infinite-dimensional) space of functions that are compact mod center. For $m \geqslant \max \left\{m_{v}: v \in\right.$ $T$ \}, we define $\mathcal{H}_{T}^{m}(\Lambda)$, respectively $\mathcal{H}_{T}^{\left(\chi_{v}\right)}(\Lambda)$ as the image of $\mathcal{H}_{T}^{a b, m} \otimes_{\mathbf{z}} \Lambda$ in the endomorphism ring of $\mathbf{C}_{\text {cusp }, T}^{\omega, m}(\Lambda)$, respectively $\mathbf{C}_{\text {cusp }, T}^{\omega,\left(\chi_{v}\right)}(\Lambda)$. Also define $\mathcal{H}_{c, T}^{m}(\Lambda)$ and $\mathcal{H}_{c, T}^{\left(\chi_{v}\right)}(\Lambda)$ as the image of $\mathcal{H}_{T}^{a b, m} \otimes_{\mathbf{z}}$ $\Lambda$ in the endomorphism ring of $\mathbf{C}_{c, T}^{\omega, m}(\Lambda)$ and $\mathbf{C}_{c, T}^{\omega,\left(\chi_{v}\right)}(\Lambda)$. Because $\mathbf{C}_{\text {cusp }, T}^{\omega, m}(\Lambda)$ is a free $\Lambda$-module of finite rank, the same holds for $\mathcal{H}_{T}^{m}(\Lambda)$ and $\mathcal{H}_{T}^{\left(\chi_{v}\right)}(\Lambda)$. Moreover $\mathcal{H}_{T}^{m}(F) \cong \mathcal{H}_{T}^{m}(\mathcal{O}) \otimes \mathcal{O} F$ and $\mathcal{H}_{T}^{\left(\chi_{v}\right)}(F) \cong \mathcal{H}_{T}^{\left(\chi_{v}\right)}(\mathcal{O}) \otimes_{\mathcal{O}} F$. (Although $\mathbf{C}_{c, T}^{\omega, m}(\Lambda)$ is not a free $\Lambda$-module of finite rank, we still have $\mathcal{H}_{c, T}^{m}(F) \cong \mathcal{H}_{c, T}^{m}(\mathcal{O}) \otimes_{\mathcal{O}} F$.)

We state the main theorem of [Laf02].

Theorem 3.6 [Laf02]. For any finite subset $T$ of $X$, there is a bijection between:

(i) smooth irreducible cuspidal automorphic representations $\Pi$ whose central character is of finite order and with $\Pi_{v}$ unramified for $v \notin\{w\} \cup T$; and

(ii) irreducible continuous representations $\rho: \pi_{1}(X \backslash(\{w\} \cup T)) \rightarrow \mathrm{GL}_{n}\left(\overline{\mathbf{Q}}_{\ell}\right)$ with determinant of finite order.

Suppose that for $\Pi$ as above, the eigenvalues of the operators $T_{v, i}, v \notin\{w\} \cup T, i=1, \ldots, n$, are $\alpha_{v, i}\left(\right.$ and $\left.\alpha_{v, 0}=1\right)$. Then the correspondence is given by the condition

$$
\operatorname{det}\left(1-x \rho\left(\operatorname{Frob}_{v}\right)\right)=\sum_{i=0}^{n} x^{n-i} \alpha_{v, i}
$$

Suppose we are given $\rho: \pi_{1}(X \backslash\{w\} \cup T) \rightarrow \mathrm{GL}_{n}(\mathcal{O})$ with absolutely irreducible residual representation $\bar{\rho}$. Let $\Pi$ be the corresponding automorphic representation. By the relation (6) the Hecke eigenvalues $\alpha_{v, i}, v \notin\{w\} \cup T, i \in 1, \ldots, n$, lie in $\mathcal{O}$, and so they define ring-homomorphisms

$$
\mathcal{H}_{v} \rightarrow \mathbf{F}: T_{v, i} \mapsto \alpha_{v, i} \quad(\bmod \mathfrak{m})
$$

for $v \notin\{w\} \cup T$.

For $v \in T$ the subspace of $U_{1, m_{v}}(v)$-invariant functions in $\Pi_{v}$ is non-trivial. If $\Pi_{v}^{U_{1, m_{v}}(v)}$ is onedimensional then $U_{0}(v)$ acts on it by a character $\chi_{v}$. If this character is non-trivial, then $\Pi_{v}$ is principal series. (If the dimension is bigger than 1 then $\Pi_{v}$ is unramified.)

If $\bar{\rho}$ is unramified at $v$ with $n$ distinct eigenvalues and if $\bar{\lambda}$ is the eigenvalue at the $(1,1)$-entry (this will be the case when we need to consider the Hecke action at T, i.e., when building TW systems), then by [HT03] one has two cases.

(1) If $\Pi_{v}$ is unramified, then $\operatorname{dim} \Pi_{v}^{U_{1, m_{v}}(v)}=n$, the action of $V_{v, n}$ on this space is semisimple, it has a unique eigenvalue which reduces to $\bar{\lambda}$ and the corresponding eigenspace is one-dimensional; this one-dimensional subspace is also an eigenspace for the operators $V_{v, i}, i=1, \ldots, n-1$. The corresponding eigenvalues are denoted $b_{v, i}$ and their mod $\mathfrak{m}$ reductions depend only on $\bar{\rho}\left(\operatorname{Frob}_{v}\right)$. 
(2) If $\Pi_{v}$ is ramified, then $\operatorname{dim} \Pi_{v}^{U_{1, m_{v}}(v)}=1$, and if we denote by $b_{v, i}$ the eigenvalues for the Hecke operators $V_{v, i}, i=1, \ldots, n$, their reductions modulo $\mathfrak{m}$ depend only on $\bar{\rho}\left(\right.$ Frob $\left._{v}\right)$ (and by the same formulas as in the first case). Moreover the reduction of $b_{v, n}$ is $\bar{\lambda}$. In either case one obtains a ring homomorphism

$$
\mathcal{H}_{v}^{m_{v}} \rightarrow \mathbf{F}: V_{v, n} \mapsto \bar{\lambda}, V_{v, i} \mapsto b_{v, i} \quad(\bmod \mathfrak{m})(i=1, \ldots, n-1)
$$

The above homomorphisms induce a ring homomorphism $\mathcal{H}_{T}^{a b, m} \rightarrow \mathbf{F}$ whose kernel is a maximal ideal which we denote by $\mathfrak{m}_{\bar{\rho}}$. This notation is justified because the homomorphism depends only on data defined in terms of $\bar{\rho}$. We also denote by $\mathfrak{m}_{\bar{\rho}}$ the image of this ideal in $\mathcal{H}_{T}^{m}(\mathcal{O}), \mathcal{H}_{T}^{\left(\chi_{v}\right)}(\mathcal{O})$, $\mathcal{H}_{c, T}^{m}(\mathcal{O})$ and $\mathcal{H}_{c, T}^{\left(\chi_{v}\right)}(\mathcal{O})$. (Since a priori there is no relation between $\bar{\rho}$ and $\mathbf{C}_{\text {cusp }, T}^{\omega, m}(\mathcal{O})$, this image can be all of $\mathcal{H}_{T}^{m}(\mathcal{O})$.) For an $\mathcal{H}_{T}^{a b, m}$-module $M$, we denote by $M_{\mathfrak{m}_{\bar{\rho}}}$ its localization at $\mathfrak{m}_{\bar{\rho}}$.

The following theorem is crucial to us, and is proved by Ogilvie [Ogi05] in forthcoming work.

Theorem 3.7 [Ogi05]. Compatible with the Hecke action of $\mathcal{H}_{T}^{a b, m}$ we have isomorphisms

$$
\mathbf{C}_{\mathrm{cusp}, T}^{\omega,\left(\chi_{v}\right)}(\mathcal{O})_{\mathfrak{m}_{\bar{\rho}}} \simeq \mathbf{C}_{c, T}^{\omega,\left(\chi_{v}\right)}(\mathcal{O})_{\mathfrak{m}_{\bar{\rho}}}
$$

and

$$
\mathbf{C}_{\mathrm{cusp}, T}^{\omega, m}(\mathcal{O})_{\mathfrak{m}_{\bar{\rho}}} \simeq \mathbf{C}_{c, T}^{\omega, m}(\mathcal{O})_{\mathfrak{m}_{\bar{\rho}}}
$$

(and the same statement after dropping the Hecke operators at $T$ ).

\subsection{Carayol's principle}

The methods of this section, which allow us to change 'types' of automorphic forms which give rise to a given $\bar{\rho}$, use a principle discovered by Carayol that occurs in the proof of [Car89, lemme 1].

We define $N_{n}(\ell)$ as the order of an $\ell$-Sylow subgroup of $\mathrm{GL}_{n}(k)$.

We may consider the Hecke action without the operators at places in $T$ and will denote the induced maximal ideal of this smaller Hecke algebra by the same symbol.

Lemma 3.8. We assume the set-up of Assumption 3.3 for sets of characters $\left(\chi_{v}\right)$ and $\left(\chi_{v}^{\prime}\right)$ such that:

(i) $\Lambda$ is the fraction field $F$ of $\mathcal{O}$;

(ii) for each $v \in T$, the product of $N_{n}(\ell)$ with the order of $\chi_{v}^{-1} \chi_{v}^{\prime}$, which we assume is a power of $\ell$, divides the order of $k_{v}^{*}$.

Then

$$
\operatorname{rank}_{\mathcal{O}} \mathbf{C}_{\text {cusp }, T}^{\omega,\left(\chi_{v}\right)}(\mathcal{O})_{\mathfrak{m}_{\bar{\rho}}}=\operatorname{rank}_{\mathcal{O}} \mathbf{C}_{\text {cusp }, T}^{\omega,\left(\chi_{v}^{\prime}\right)}(\mathcal{O})_{\mathfrak{m}_{\bar{\rho}}}
$$

(and the same conclusion holds if we consider the corresponding maximal ideal of the smaller Hecke algebra without the operators at the places in $T$ ).

Localization at $\mathfrak{m}_{\bar{\rho}}$ commutes with reduction $\mathcal{O} \rightarrow \mathbf{F}$ of the ring of integers of $F$ to its residue field. Furthermore, after reduction one has $\chi_{v} \equiv \chi_{v}^{\prime} \equiv 1$ in $\mathbf{F}$ for all $v \in T$. So in view of Proposition 3.5(i)(ii) and Theorem 3.7 due to Ogilvie, it suffices to prove the following lemma.

Lemma 3.9. Suppose the hypotheses of Lemma 3.8 hold. Then the submodules $\mathbf{C}_{c, T}^{\omega,\left(\chi_{v}\right)}(\mathcal{O}) \otimes_{\mathcal{O}} \mathbf{F}$ and $\mathbf{C}_{c, T}^{\omega,\left(\chi_{v}^{\prime}\right)}(\mathcal{O}) \otimes_{\mathcal{O}} \mathbf{F}$ of $\mathbf{C}_{c, T}^{\omega, m}(\mathbf{F})$ agree.

Proof. By $Z$ we denote the center of $\mathrm{GL}_{n}$, viewed as an algebraic group over $K$, and we regard $Z_{T}^{m}$ as a subgroup of $Z(\mathbf{A})$. Because of the central action and the conditions on the places $v \in T$, elements in $\mathbf{C}_{c, T}^{\omega,\left(\chi_{v}\right)}(\mathcal{O})$ may be thought of as functions with finite support and values in $\mathcal{O}$ on the 


\section{G. BöCKLE AND C. Khare}

infinite set $\mathrm{GL}_{n}(K) Z_{T}^{m} \backslash \mathrm{GL}_{n}(\mathbf{A}) / U_{T}^{0}$. Choose $g_{j} \in \mathrm{GL}_{n}(\mathbf{A}), j \in J$, such that

$$
\mathrm{GL}_{n}(\mathbf{A})=\coprod_{j \in J} \operatorname{GL}_{n}(K) Z_{T}^{m} g_{j} U_{T}^{0} .
$$

Let $\mathcal{O}\left(\left(\chi_{v}\right)\right)$ denote the representation of $Z_{T}^{m} U_{T}^{0} / Z(K) U_{T}^{m}$ on $\mathcal{O}$ given by the (compatible) action of $\omega$ and of the $\chi_{v}$. Then $\mathbf{C}_{c, T}^{\omega,\left(\chi_{v}\right)}(\mathcal{O})$ can also be identified with elements of the direct sum $\bigoplus_{j \in J} \mathcal{O}\left(\left(\chi_{v}\right)\right)^{\mathrm{GL}_{n}(K) \cap Z_{T}^{m} g_{j}^{-1} U_{T}^{0} g_{j}}$, because in addition to the actions by $\omega$ and the $\chi_{v}$, the component at $g_{j}$ also carries a trivial action by $\mathrm{GL}_{n}(K)$. (We have analogous descriptions for forms with $\mathbf{F}$-coefficients, and for the space $\mathbf{C}_{c, T}^{\omega,\left(\chi_{v}^{\prime}\right)}(\Lambda)$ with $\Lambda \in\{\mathcal{O}, \mathbf{F}\}$.)

Since we prefer to work with the action of the finite group $Z_{T}^{m} / Z(K) \pi_{w}^{\mathbf{Z}}$, we observe that elements in $\mathbf{C}_{c, T}^{\omega, \ldots}(\ldots)$ may be regarded as functions on the set $\mathrm{GL}_{n}(\mathbf{A}) / Z(K) \pi_{w}^{\mathbf{Z}}$. By Lemma 3.10 below, the $\ell$-part of the exponents of (the finite groups) $\left(\mathrm{GL}_{n}(K) \pi_{w}^{\mathbf{Z}} \cap Z_{T}^{m} g_{j}^{-1} U_{T}^{0} g_{j}\right) / Z(K) \pi_{w}^{\mathbf{Z}}$ divide $N_{n}(\ell)$ for all $j$. Using the second hypothesis of Lemma 3.8 we have

$$
\mathcal{O}\left(\left(\chi_{v}\right)\right)^{\mathrm{GL}_{n}(K) \cap Z_{T}^{m} g_{j} U_{T}^{0} g_{j}^{-1}} \cong \mathcal{O}\left(\left(\chi_{v}^{\prime}\right)\right)^{\mathrm{GL}_{n}(K) \cap Z_{T}^{m} g_{j}^{-1} U_{T}^{0} g_{j}}
$$

for all $j \in J$. Hence for each $j \in J$ either this set is zero, or agrees with $\mathcal{O}$. This completes the proof of Lemma 3.9.

Lemma 3.10. If $\gamma \in \mathrm{GL}_{n}(K) / K^{*}$ is of $\ell$-power order, its order divides $N_{n}(\ell)$.

Proof. We have the exact sequence

$$
0 \longrightarrow \mathrm{SL}_{n}(K) /\left\{x \in K^{*}: x^{n}=1\right\} \longrightarrow \mathrm{GL}_{n}(K) / K^{*} \longrightarrow K^{*} / K^{* n} \longrightarrow 0 .
$$

Because the image of $\gamma$ in $K^{*} / K^{* n}$ is of $\ell$-power order, it must lie in $k^{*} / k^{* n}$. Because $\ell$ is prime to $n$, the order of this image divides the order of the $\ell$-Sylow of $k^{*}$. Say the latter order is $n_{\ell}$. Then $\gamma^{n_{\ell}}$ lies in $\mathrm{SL}_{n}(K) /\left\{x \in K^{*}: x^{n}=1\right\}$. Again because $n$ is prime to $\ell$, there is an element $\gamma^{\prime} \in \mathrm{SL}_{n}(K)$ which maps to $\gamma^{n_{\ell}}$ and whose order is the same as that of $\gamma^{n_{\ell}}$. Thus it suffices to show that, if $\gamma^{\prime} \in \mathrm{SL}_{n}(K)$ is of $\ell$-power order $\ell^{d}$, then the order of $\gamma^{\prime}$ divides the order of an $\ell$-Sylow of $\mathrm{SL}_{n}(k)$.

Let $k^{\prime}$ be the smallest extension of $k$ which contains a primitive $\ell^{d}$ th root of unity. We assume that $\gamma^{\prime}$ is given in rational canonical form over $K$. We claim that $\gamma^{\prime}$ has entries in $k$. For this we may assume that the rational canonical form consists of a single block, which is thus completely determined by the characteristic polynomial of $\gamma^{\prime}$. But the characteristic polynomial has coefficients in $k^{\prime}$ and in $K$ and thus in $k$. Hence $\gamma^{\prime}$ has coefficients in $k$. Thus $\gamma^{\prime}$ lies up to conjugation in $\operatorname{SL}_{n}(k)$, and our last assertion is shown.

\subsection{Taylor-Wiles systems}

Throughout this section, we fix a lift $\rho: \pi_{1}(X) \rightarrow \mathrm{GL}_{n}(\mathcal{O})$ of $\bar{\rho}$ and make the following assumption.

Assumption 3.11. We assume that $\eta:=\operatorname{det} \rho$ is of finite order.

For each $m \in \mathbf{N}$ we also fix a finite subset $Q_{m} \subset X \backslash\{w\}$ of places such that for all $v \in Q_{m}$ the matrix $\bar{\rho}\left(\operatorname{Frob}_{v}\right)$ has distinct eigenvalues and $q_{v} \equiv 1\left(\bmod \ell^{m}\right)$. In this section we will complement the Galois-theoretic work in $\S 2$ by automorphic results that together yield the existence of TaylorWiles systems.

Define $\Delta_{m}$ as the maximal quotient of $\prod_{v \in Q_{m}} k_{v}^{*}$ of exponent $\ell^{m}$. Via the projection onto the $(1,1)$-entry, we identify

$$
\Delta_{m} \cong \prod_{v \in Q_{m}} U_{0}(v) / U_{1, m}(v)
$$


Mod $\ell$ REPRESENTATIONS OF ARITHMETIC FUNDAMENTAL GROUPS, II

There is a natural ('diamond') action of $\Delta_{m}$ on the spaces $\mathbf{C}_{\text {cusp }, Q_{m}}^{\omega, m}(\mathcal{O})_{\mathfrak{m}_{\bar{\rho}}}$ and $\mathbf{C}_{c, Q_{m}}^{\omega, m}(\mathcal{O})_{\mathfrak{m}_{\bar{\rho}}}$. Using Lemma 3.9 and its proof, we easily show the following result.

Proposition 3.12. We fix a positive integer $m$ and assume the set-up of Assumption 3.3, with

(a) the set of places $Q_{m}$ here playing the role of $T$ there,

(b) $\Lambda$ a discrete valuation ring $\mathcal{O}$ containing $W(\mathbf{F})\left[\zeta_{\ell^{m}}\right]$, and

(c) $\ell^{m} N_{n}(\ell)$ dividing the order of $k_{v}^{*}$ for all $v \in Q_{m}$.

Then $\mathbf{C}_{\text {cusp, } Q_{m}}^{\omega, m}(\mathcal{O})_{\mathfrak{m}_{\bar{\rho}}}$ is free over $\mathcal{O}\left[\Delta_{m}\right]$, and for the invariants under $\Delta_{m}$ one has

$$
\left(\mathbf{C}_{\text {cusp }, Q_{m}}^{\omega, m}(\mathcal{O})_{\mathfrak{m}_{\bar{\rho}}}\right)^{\Delta_{m}}=\mathbf{C}_{\text {cusp }, Q_{m}}^{\omega, 0}(\mathcal{O})_{\mathfrak{m}_{\bar{\rho}}} .
$$

Note that by Proposition 3.4, one has the isomorphism

$$
\mathbf{C}_{\mathrm{cusp}, Q_{m}}^{\omega, m}(\mathcal{O})_{\mathfrak{m}_{\bar{\rho}}} \otimes F \cong \bigoplus_{\left(\chi_{v}^{\prime}\right)} \mathbf{C}_{\text {cusp }, Q_{m}}^{\omega,\left(\chi_{v}^{\prime}\right)}(\mathcal{O})_{\mathfrak{m}_{\bar{\rho}}} \otimes F
$$

where the sum is over all characters of $\left(\chi_{v}^{\prime}\right)$ of $\Delta_{m}$.

Proof. The second assertion is obvious from the definitions. To prove the first, observe that because localization at $\mathfrak{m}_{\bar{\rho}}$ commutes with the action of $\Delta_{m}$ and because of Theorem 3.7, it will suffice to show that $\mathbf{C}_{c, Q_{m}}^{\omega, m}(\mathcal{O})$ is free over $\mathcal{O}\left[\Delta_{m}\right]$. This follows easily (see proof of Proposition 5.6.1 of [CDT99]) from the fact that, for all characters $\left(\chi_{v}\right)$ of $\Delta_{m}$, the rank of $\mathbf{C}_{c, Q_{m}}^{\omega,\left(\chi_{v}\right)}(\mathcal{O})_{\mathfrak{m}_{\bar{\rho}}} \otimes F$ is independent of $\left(\chi_{v}\right)$ as follows from Lemma 3.8.

We now define universal deformation and Hecke rings corresponding to the above situation.

Choose for each $v \in Q_{m}$ a Teichmüller lift $\lambda_{v}$ of one of the eigenvalues of $\bar{\rho}\left(\right.$ Frob $\left._{v}\right)$. Write $\underline{\lambda}$ for $\left(\lambda_{v}\right)_{v \in Q_{m}}$. Let

$$
\rho_{X, \bar{Q}_{m}}^{m, \frac{\lambda}{2}}: \pi_{1}\left(X \backslash\left(\{w\} \cup Q_{m}\right)\right) \rightarrow \mathrm{GL}_{n}\left(R_{X, \bar{Q}_{m}}^{m, \lambda}\right)
$$

denote the universal deformation that parameterizes deformations $\tilde{\rho}: \pi_{1}\left(X \backslash\left(\{w\} \cup Q_{m}\right)\right) \rightarrow \mathrm{GL}_{n}(R)$, $R \in \mathcal{A}, R$ an $\mathcal{O}$-algebra, of $\bar{\rho}$ such that:

(i) $\operatorname{det} \tilde{\rho}_{w}$ factors via $G_{w} \stackrel{s_{w}}{\longrightarrow} \bar{I}_{w}$ and $\tilde{\rho}_{w} \otimes\left(\operatorname{det} \tilde{\rho}_{w}\right)^{-1 / n}$ is unramified; and

(ii) for all $v \in Q_{m}, \rho_{v}$ is of type $\mathcal{C}_{v, \lambda_{v}, m}$ as defined above Lemma 2.8.

Note also that from the local conditions at $v \in Q_{m}$, via the action of $I_{v}$ one obtains a homomorphism $\mathcal{O}\left[\Delta_{m}\right] \rightarrow R_{X, \bar{Q}_{m}}^{m, \lambda}$

We define $\omega: \mathrm{GL}_{1}(\mathbf{A}) \rightarrow \mathcal{O}^{*}$ as the Hecke character corresponding to $\eta=\operatorname{det} \rho$. For $v \in T$ we let $\chi_{v}: k_{v}^{*} \rightarrow \mathcal{O}^{*}$ be the trivial character. Then the above data satisfies Assumption 3.3 for $\Lambda=\mathcal{O}$. Suppose a basis of $A_{v}^{n}$ is chosen in such a way that $e_{1, v}$ corresponds to the eigenvalue $\lambda_{v}$. We now define

$$
\mathbf{T}_{X, Q_{m}}^{m, \lambda}:=\left(\mathcal{H}_{Q_{m}}^{m}(\mathcal{O})\right)_{\mathfrak{m}_{\bar{\rho}}} .
$$

Let $\tau_{1}, \ldots, \tau_{s}$ be a list of the Galois representations corresponding via Lafforgue's theorem to eigenforms in $\widetilde{\mathbf{C}}_{S, Q_{m}}^{\omega, m}(\mathcal{O})_{\mathfrak{m}_{\bar{\rho}}}$. By choice of the maximal ideal and definition of the Hecke action at places in $Q_{m}$, using the $\mathbf{C}$-valued theory one finds that the algebra $\mathbf{T}_{X, Q_{m}}^{m, \lambda} \otimes_{\mathcal{O}} F$ is semisimple, cf. [HT03, III.2, second paragraph]. We therefore denote by

$$
\tau:=\tau_{1} \oplus \cdots \oplus \tau_{s}: \pi_{1}\left(X \backslash Q_{m}\right) \longrightarrow \mathrm{GL}_{n}\left(\mathbf{T}_{X, \bar{Q}_{m}}^{m, \lambda} \otimes_{\mathcal{O}} F\right)
$$

the corresponding Galois representation. 


\section{G. BÖCKLE AND C. Khare}

Proposition 3.13. The representation $\tau$ can be factored as a composition

$$
\pi_{1}\left(X \backslash Q_{m}\right) \stackrel{\tau_{X, \bar{Q}_{m}}^{m, \lambda}}{\longrightarrow} \mathrm{GL}_{n}\left(\mathbf{T}_{X, \bar{Q}_{m}}^{m, \lambda}\right) \hookrightarrow \mathrm{GL}_{n}\left(\mathbf{T}_{X, \bar{Q}_{m}}^{m, \lambda} \otimes_{\mathcal{O}} F\right) .
$$

Proof. Because $\bar{\rho}$ is absolutely irreducible, by a result of Carayol [Car94] the image of $\tau$ lies in the ring of traces. By the Čebotarev density theorem, this ring is spanned by the coefficients of the characteristic polynomials of

$$
\tau\left(\operatorname{Frob}_{v}\right), \quad v \notin\{w\} \cup Q_{m} .
$$

Thus by Lafforgue's theorem, it is spanned by the Hecke eigenvalues of the corresponding eigenforms. Thus the ring of traces lies in $\mathbf{T}_{X, \bar{Q}_{m}}^{m, \lambda}$.

From the definition of $\tau_{X, \bar{Q}_{m}}^{m, \lambda}$ it is clear that it is a representation of the type parameterized by $\rho_{X, Q_{m}}^{m, \frac{\lambda}{2}}$. By universality there arises a unique morphism $R_{X, Q_{m}}^{m, \frac{\lambda}{Q_{m}}} \rightarrow \mathbf{T}_{X, \bar{Q}_{m}}^{m, \lambda}$ such that $\tau_{X, \bar{Q}_{m}}^{m, \lambda}$ is induced from $\rho_{X, Q_{m}}^{m, \lambda}$. Both rings are non-zero because of the existence of $\rho$.

Proposition 3.14. The induced morphism $R_{X, \bar{Q}_{m}}^{m, \frac{\lambda}{Q}} \rightarrow \mathbf{T}_{X, \bar{Q}_{m}}^{m, \lambda}$ is surjective.

Proof. By Nakayama's lemma it suffices to prove the assertion modulo $\mathfrak{m}$. It is clear from the Langlands correspondence that the reductions modulo $\mathfrak{m}$ of the Hecke operators $T_{v, i}, v \notin\{w\} \cup T$, and $T_{w, i}^{\prime}, i=1, \ldots, n$, lie in the image. At places $v \in T$ this again follows from the compatibility of the global Langlands correspondence with the local one, and the explicit decription of the Hecke action at places in $T$, cf. [HT03, V.1.5]. Namely, the action of $V_{v, n}(\bmod \mathfrak{m})$ is given by the first eigenvalue $\bar{\lambda}$ of $\bar{\rho}\left(\operatorname{Frob}_{v}\right)$ and the action of $V_{v, i}(\bmod \mathfrak{m}), i=1, \ldots, n-1$, by the elements $b_{v, i}(\bmod \mathfrak{m})$ which are expressions in the elementary symmetric polynomials in the remaining eigenvalues of $\bar{\rho}\left(\operatorname{Frob}_{v}\right)$. So in this case, too, the reductions of the Hecke operators lie in the image of $R_{X, \bar{Q}_{m}}^{m,}$.

If $Q_{m}=\emptyset$, we drop it as well as $m$ and $\underline{\lambda}$ from the notation, and add a superscript zero, i.e. the above morphism becomes $R_{X}^{0} \rightarrow \mathbf{T}_{X}^{0}$. We have the following central result.

Theorem 3.15. Suppose that:

(i) $\eta$ is of finite order (Assumption 3.11);

(ii) for any $\pi_{1}(X)$-subrepresentation $V$ of $\operatorname{ad}(\bar{\rho})$, there exists a regular semisimple $g_{V} \in \bar{\rho}\left(\pi_{1}(\bar{X})\right)$ such that $V^{g_{V}} \neq 0$;

(iii) if $\zeta_{\ell} \in K$, then $\operatorname{ad}^{0}(\bar{\rho})$ has no one-dimensional subrepresentation;

(iv) if $\zeta_{\ell} \in E$, then $H^{1}\left(\operatorname{Gal}\left(E / K\left(\zeta_{\ell}\right)\right), \operatorname{ad}^{0}(\bar{\rho})\right)=0$;

(v) $\operatorname{im}(\bar{\rho})$ contains no normal subgroup of index $\ell$.

Then $R_{X}^{0} \rightarrow \mathbf{T}_{X}^{0}$ is an isomorphism.

Proof. The proof is based on the use of Taylor-Wiles systems in the improved form due independently to Diamond and Fujiwara, cf. [Dia97] and [Fuj]. For each $m \in \mathbf{N}$, use Lemma 2.9 to choose a set $Q_{m} \subset X$ such that

(a) $\# Q_{m}=d:=\operatorname{dim} H_{\left\{L_{v}\right\}}^{1}(\{w\}, \operatorname{ad}(\bar{\rho})(1))$,

(b) $q_{v} \equiv 1\left(\bmod N_{n}(\ell) \ell^{m}\right)$ for all $v \in Q_{m}$,

(c) $\bar{\rho}\left(\right.$ Frob $\left._{v}\right)$ has distinct eigenvalues for each $v \in Q_{m}$, and

(d) $H_{\left\{L_{v} \frac{1}{v}\right.}^{1}\left(\{w\} \cup Q_{m}, \operatorname{ad}(\bar{\rho})\right)=0$ where $L_{v}=L_{v, \lambda_{v}}$ for each $v \in Q_{m}$ and $\lambda_{v}$ is the Teichmüller lift of some eigenvalue of $\bar{\rho}\left(\right.$ Frob $\left._{v}\right)$. 
Note that $\operatorname{dim} H_{\left\{L_{v}\right\}}^{1}(\{w\}, \operatorname{ad}(\bar{\rho}))=\operatorname{dim} H_{\left\{L_{v}\right\}}^{1}\left(\{w\} \cup Q_{m}, \operatorname{ad}(\bar{\rho})\right)$, and define $\underline{\lambda}_{m}$ as $\left(\lambda_{v}\right)_{v \in Q_{m}}$.

We introduce notation similar to [Dia97, $\S 2]$. Define

$$
\begin{aligned}
\mathbf{R} & :=R_{X}^{0} / \mathfrak{m}, \\
\mathbf{T} & :=\mathbf{T}_{X}^{0} / \mathfrak{m}, \\
\mathbf{R}_{m} & :=R_{X, Q_{m}}^{m, \lambda_{m}} / \mathfrak{m} \\
\mathbf{T}_{m} & :=\mathbf{T}_{X, \underline{Q}_{m}}^{m, \underline{\lambda}_{m}} / \mathfrak{m} \\
\mathbf{H} & :=\operatorname{Hom}_{\mathcal{O}}\left(\widetilde{\mathbf{C}}_{S, \emptyset}^{0, \omega}(\mathcal{O})_{\mathfrak{m}_{\bar{\rho}}}, \mathbf{F}\right), \\
\mathbf{H}_{m} & :=\operatorname{Hom}_{\mathcal{O}}\left(\widetilde{\mathbf{C}}_{S, Q_{m}}^{m, \omega}(\mathcal{O})_{\mathfrak{m}_{\bar{\rho}}}, \mathbf{F}\right), \\
\mathbf{A}_{m} & :=\mathcal{O}\left[\Delta_{m}\right] / \mathfrak{m} \cong \mathbf{F}\left[\left[y_{1}, \ldots, y_{d}\right]\right] /\left(y_{1}, \ldots, y_{d}\right)^{m} .
\end{aligned}
$$

One easily verifies from the preceding work that:

(i) each $\mathbf{R}_{m}$ is topologically generated by $d$ elements over $\mathbf{F}$;

(ii) $\mathbf{R}_{m} / \mathfrak{m}_{\mathbf{R}_{m}}^{m} \cong \mathbf{F}\left[\left[x_{1}, \ldots, x_{d}\right]\right] /\left(x_{1}, \ldots, x_{d}\right)^{m}$;

(iii) there exists a canonical $\mathbf{R}_{m}$-linear surjection $\pi_{m}: \mathbf{H}_{m} \longrightarrow \mathbf{H}$;

(iv) under $\mathbf{R}_{m} \longrightarrow \mathbf{R}$ the image of $\left(y_{1}, \ldots, y_{d}\right)$ is zero;

(v) $\mathbf{H}_{m}$ is via $\mathbf{A}_{m} \rightarrow \mathbf{R}_{m} \rightarrow \mathbf{T}_{m}$ a module over $\mathbf{A}_{m}$ and $\mathbf{R}_{m}$ and the action of $\mathbf{A}_{m}$ is the same as that which occurs in Proposition 3.12;

(vi) $\mathbf{H}_{m}$ is free over $\mathbf{A}_{m}$ (by Proposition 3.12).

For instance, part (i) follows from Lemma 2.9(d) and the choice of $Q_{m}$. Part (ii) follows from Lemma 2.9(c), which expresses that all relations are local, and from definition (3) of the local ring $R_{v, m}$, which shows that the local relations at the places in $Q_{m}$ are trivial modulo the $m$ th power of the maximal ideal. Part (iii) is a trivial consequence of Proposition 3.12. Part (iv) is clear since deformations parameterized by $\mathbf{R}$ are unramified at the places in $Q_{m}$ while the variables $y_{1}, \ldots, y_{d}$ describe the ramification at $Q_{m}$ of deformations parameterized by $\mathbf{R}_{m}$.

We now verify the following assertion that is also a crucial part of constructing Taylor-Wiles systems:

(vii) the morphism $\pi_{m}$ induces an isomorphism $\mathbf{H}_{m} /\left(y_{1}, \ldots, y_{d}\right) \cong \mathbf{H}$ where we consider these as modules over $R_{X, \underline{Q}_{m}}^{m, \lambda_{m}}$.

In view of Proposition 3.12 and the above definitions it suffices to show that we have an isomorphism

$$
\mathbf{C}_{\text {cusp }, \emptyset}^{\omega, 0}(\mathcal{O})_{\mathfrak{m}_{\bar{\rho}}} \simeq \mathbf{C}_{\text {cusp }, Q_{m}}^{\omega, 0}(\mathcal{O})_{\mathfrak{m}_{\bar{\rho}}}
$$

as modules over $R_{X, Q_{m}}^{m, \lambda_{m}}$. This follows from the arguments in the proofs of [HT03, Propositions V.2.3 and V.2.4], and where the isomorphism above is given by the (exact analog of the) map $X_{\infty, Q_{m}}$ of [HT03]. We give some details. As remarked in [HT03] we need to prove the isomorphism in (7) only after tensoring with $\overline{\mathbf{Q}}_{\ell}$. We first prove that all forms that contribute to the right-hand side are old at places in $Q_{m}$.

For this let $f$ be a cuspidal Hecke eigenform for $\mathrm{GL}_{n}$ whose image in the right-hand side of (7) is non-trivial. Let $v$ be in $Q_{m}$. As the $\ell$-adic representation $\rho_{f}$ corresponding to $f$ reduces to $\bar{\rho}$ residually, $\rho_{f}$ restricted to a decomposition group $G_{v}$ at $v$ is a lift of $\bar{\rho}_{v}$ to $\mathcal{O}$. By Lemma 2.7 such lifts are diagonalizable and therefore finite on inertia. Hence by the compatibility of the global Langlands correspondence of [Laf02] with the local Langlands correspondence and the unramifiedness of the central character at $v, \Pi_{v}(f)$ is an unramified principal series representation. 


\section{G. BÖCKLE AND C. Khare}

We are now in the situation described after Theorem 3.6 and quoted from [HT03]. The action of $V_{v, n}$ on $\Pi_{v}(f)^{U_{0}(v)}$ is diagonalizable and there is exactly one eigenvalue whose reduction modulo $\mathfrak{m}$ is $\lambda_{v}(\bmod \mathfrak{m})$, and it has multiplicity one. Therefore after localization at $\mathfrak{m}_{\bar{\rho}}$ at most a onedimensional subspace of $\Pi_{v}(f)^{U_{0}(v)}$ remains. That the remaining space is indeed one-dimensional follows from the fact that (7) is injective. We have thus completed the proof of (vii).

It now follows from [Dia97, Theorem 2.1] that $\mathbf{R}$ is a complete intersection of dimension zero and $\mathbf{H}$ is free over $\mathbf{R}$. Since the action of $\mathbf{R}$ on $\mathbf{H}$ factors via $\mathbf{T}$, it follows in particular that $\mathbf{R} \rightarrow \mathbf{T}$ must be injective and hence an isomorphism.

Let us now come back to the original question. Because $\mathbf{T}_{X, \bar{Q}_{m}}^{m, \lambda}$ is $\mathcal{O}$-torsion free and finitely generated, the surjection $R_{X, \bar{Q}_{m}}^{m, \lambda} \longrightarrow \mathbf{T}_{X, \bar{Q}_{m}}^{m, \lambda}$ splits as a map of $\mathcal{O}$-modules. By the above its reduction modulo $\mathfrak{m}$ is an isomorphism. But then the morphism itself must be bijective. The proof of Theorem 3.15 is now complete.

\subsection{Lowering the level $\grave{a}$ la Skinner and Wiles}

We have the following 'lowering the level' result as in the work of Skinner and Wiles [SW01].

Theorem 3.16. Suppose $\rho: \pi_{1}(X \backslash T) \rightarrow \operatorname{GL}_{n}(\mathcal{O})$ and $\bar{\rho}:=\rho(\bmod \mathfrak{m})$ satisfy the following conditions:

(i) $\mathcal{O}$ contains $\zeta_{\ell}$;

(ii) for all $v \in T, \rho$ is type- 1 and $\ell N_{n}(\ell)$ divides the order of $k_{v}^{*}$.

Then there exists a representation $\rho^{\prime}: \pi_{1}(X \backslash T) \rightarrow \mathrm{GL}_{n}(\mathcal{O})$ such that:

(i) the residual representations of $\rho$ and $\rho^{\prime}$ agree;

(ii) $\rho^{\prime}\left(I_{v}\right)$ is finite for $v \in T$.

Remark 3.17. This theorem is referred to as a level lowering result as from it one deduces that there is a solvable base change $Y \rightarrow X$, that one can make disjoint from any given covering of $X$, such that $\left.\rho^{\prime}\right|_{\pi_{1}(Y)}$ has conductor the conductor of $\left.\bar{\rho}\right|_{\pi_{1}(Y)}$. We use this in the section that is coming up!

Proof of Theorem 3.16. We use Lafforgue's theorem to convert the above into an assertion about cusp eigenforms that we have proved in Lemma 3.8. Via Lafforgue's theorem, which is compatible with the local Langlands correspondence, $\rho$ corresponds to a cuspidal Hecke eigenform in $\mathbf{C}_{\text {cusp }, T}^{\omega,\left(\chi_{v}\right)}(\mathcal{O})_{\mathfrak{m}_{\bar{\rho}}} .($ In this proof we do not need to consider Hecke action at places in $T$.)

Let now $\left(\chi_{v}^{\prime}\right)$ be such that $\chi_{v}^{\prime}$ is of exact order $\ell$ at all $v \in T$. By Lemma 3.8 we find a non-zero cuspidal Hecke eigenform $f^{\prime}$ in $\mathbf{C}_{\text {cusp }, T}^{\omega,\left(\chi_{v}^{\prime}\right)}(\mathcal{O})_{\mathfrak{m}_{\bar{\rho}}}$. Let $\rho^{\prime}$ be the corresponding Galois representations, which exist by Lafforgue's theorem. The first assertion is clear from the definition of $\mathfrak{m}_{\bar{\rho}}$. At places in $T$, it follows from the non-triviality of $\chi_{v}^{\prime}$ that $\Pi_{v}\left(f^{\prime}\right)$ is ramified principal series (as recalled just before Theorem 3.7 above). This shows the second assertion.

\section{Proof of main theorems}

We can finally give the proof of our central theorem.

Proof of Theorem 3.1. We argue by contradiction, and, in view of Theorem 1.3, assume that there is a representation $\rho^{\prime}: \pi_{1}(X) \rightarrow \mathrm{GL}_{n}(\mathbf{F}[[x]])$ with $\bar{\rho}=\rho^{\prime}(\bmod x)$ and $\rho^{\prime}\left(\pi_{1}(\bar{X})\right)$ infinite. Because $\ell$ does not divide $n$, taking $n$th roots is an isomorphism on the 1-units in $\mathbf{F}[[x]]^{*}$. Therefore the $n$th root of the 1-unit part of $\operatorname{det} \rho^{\prime}$ is a character, say $\tilde{\eta}$, of $\pi_{1}(X)$. It has the $\operatorname{property} \operatorname{det}\left(\tilde{\eta} \cdot \rho^{\prime}\right)=\operatorname{det} \bar{\rho}$. Since the image of $\pi_{1}(\bar{X})$ under any character of $\pi_{1}(X)$ is finite, we will from now on assume $\operatorname{det} \rho^{\prime}=\operatorname{det} \bar{\rho}$. 
We now consider the ring $R:=\operatorname{ker}(\mathcal{O} \oplus \mathbf{F}[[x]] \longrightarrow \mathbf{F})$. It lies in $\mathcal{A}$ and affords a representation $\rho^{\prime \prime}:=\rho \oplus \rho^{\prime}: \pi_{1}(X) \rightarrow \mathrm{GL}_{n}(R)$ with determinant $\eta$. Set $T_{i}:=T$ the set of places at which $\rho$ is type-1. Take $m \in \mathbf{N}$ such that $\ell N_{n}(\ell)$ divides $\# k_{w, m}^{*}$ for all $w \in T_{i}$. Then Corollary 2.5 applied to $\rho^{\prime \prime}$ provides us with a finite Galois covering $Y \rightarrow X$ such that:

(a) $Y$ is geometrically connected over $k$;

(b) $\rho^{\prime \prime}\left(\pi_{1}(Y)\right)=\rho^{\prime \prime}\left(\pi_{1}(X)\right), \bar{\rho}\left(\pi_{1}(\bar{Y})\right)=\bar{\rho}\left(\pi_{1}(\bar{X})\right)$;

(c) $\rho$ is unramified at places not above $T_{i}$;

(d) $\rho_{\mid G_{w^{\prime}}}$ is type- 1 and $\ell \cdot N_{n}(\ell) \mid \# k_{w^{\prime}}^{*}$ for all $w^{\prime}$ above a place $w \in T_{i}$.

Since $\rho_{\pi_{1}(Y)}$ satisfies all the conditions originally imposed on $\rho$, we may therefore rename $Y$ to $X$, assume that $\rho^{\prime}$ is unramified everywhere and has determinant $\operatorname{det} \bar{\rho}$, and that $\rho$ satisfies the following conditions:

(i) $\bar{\rho}:=\rho(\bmod \mathfrak{m})$ is absolutely irreducible;

(ii) $\bar{\rho}\left(\pi_{1}(\bar{X})\right)$ contains a regular semisimple element;

(iii) $\eta:=\operatorname{det} \rho$ is of finite order;

(iv) at places $v$ at which $\rho$ ramifies, $\rho_{v}$ is type- 1 and $\ell N_{n}(\ell)$ divides $\# k_{v}^{*}$.

Condition (iv) allows us to apply Theorem 3.16 on level lowering. Thereby we may replace (iv) by:

$\left(\right.$ iv $\left.^{\prime}\right) \rho\left(I_{v}\right)$ is finite at all places where $\rho$ ramifies and $\bar{\rho}$ does not.

By yet another application of Corollary 2.5 to $\rho^{\prime \prime}$ with $T_{i}=\emptyset$, the latter condition may be replaced by:

$\left(\right.$ iv $\left.{ }^{\prime \prime}\right) \rho^{\prime \prime}$ is unramified at all places.

Recall that $R_{X}^{0}$ is universal for deformations of $\bar{\rho}$ to $\mathcal{O}$-algebras in $\mathcal{A}$ which are unramified outside $\{w\}$, and unramified at $w$ after a twist by a character of order $\ell$ that factors via $s_{w}$. Because $\rho^{\prime}$ is unramified everywhere, there is a unique morphism $\phi: R_{X}^{0} \rightarrow \mathbf{F}[[x]]$ which induces $\rho^{\prime}$. The ring $\mathbf{F}[[x]]$ is of characteristic $\ell$ and so $\phi$ factors via $R_{X}^{0} /(\ell)$.

On the other hand by Theorem 3.15, which used the technique of Taylor-Wiles systems, the ring $R_{X}^{0}$ is finite over $\mathbf{Z}_{\ell}$. Therefore $R_{X}^{0} /(\ell)$ is finite, and this contradicts our assumption that $\rho^{\prime}$ has infinite image.

When combined with Lemma 2.11 and [BK05, Proposition 2.6], the following result implies Theorem 1.6. (Note that (i) below implies $\ell \not n n$, and that we are assuming that $X$ is projective which implies that in the situation of Theorem 1.6 the lifting result of [BK05] is available.)

Theorem 4.1. Suppose that $\bar{\rho}: \pi_{1}(X) \rightarrow \mathrm{GL}_{n}(\mathbf{F})$ satisfies the following:

(i) $\operatorname{ad}^{0}(\bar{\rho})$ is absolutely irreducible over $\mathbf{F}_{\ell}[\operatorname{im}(\bar{\rho})]$;

(ii) if $\zeta_{\ell} \in E$, then $H^{1}\left(\operatorname{Gal}\left(E / K\left(\zeta_{\ell}\right)\right), \operatorname{ad}^{0}(\bar{\rho})\right)=0$ and $\operatorname{ad}^{0}(\bar{\rho})$ is absolutely irreducible over $\mathbf{F}\left[\bar{\rho}\left(\pi_{1}(Z)\right)\right]$, where $Z \rightarrow X$ corresponds to $K\left(\zeta_{\ell}\right) / K$;

(iii) $\bar{\rho}$ admits $R$-places;

(iv) $\bar{\rho}\left(\pi_{1}(\bar{X})\right)$ contains a regular semisimple element.

Then $R_{\bar{\rho}}^{\eta}$ is finite over $\mathbf{Z}_{\ell}$, where $\eta$ is the Teichmüller lift of $\operatorname{det} \bar{\rho}$.

Proof. To prove the assertion on $R_{X}^{\eta}$ we may, as in the preceding proof, pass from $X$ to a finite Galois cover provided that we preserve all our original hypotheses. 


\section{G. Böckle And C. Khare}

Using (i)-(iii) we obtain from [BK05, Theorem 2.4] a finite set $T \subset X$ and a representation $\rho: \pi_{1}(X \backslash T) \rightarrow \mathrm{GL}_{n}(W(\mathbf{F}))$ such that $\rho$ is type-1 at places in $T$.

Suppose now that $\operatorname{im}(\bar{\rho})$ contains a normal subgroup of index $\ell$, and let $\pi: Y \rightarrow X$ be the corresponding Galois cover of degree $\ell$. Because $\ell \backslash n$, the modular representation theory of finite groups shows that $\operatorname{ad}^{0}(\bar{\rho})$ is still absolutely irreducible over $\bar{\rho}\left(\pi_{1}(Y)\right)$. Also (iv) still holds for $\left.\bar{\rho}\right|_{\pi_{1}(Y)}$. We claim that (ii) still holds for $\bar{\rho}_{\mid \pi_{1}(Z)}$.

So suppose $\zeta_{\ell} \in E$. By the reasoning given above, $\operatorname{ad}^{0}(\bar{\rho})$ will still be absolutely irreducible over $\mathbf{F}\left[\pi_{1}\left(Z_{Y}\right)\right]$ for the pullback $Z_{Y} \rightarrow Y$ of $Z \rightarrow X$ along $Y \rightarrow X$. Let $K^{\prime} / K\left(\zeta_{\ell}\right)$ be the field extension corresponding to $Z_{Y} \rightarrow Y$. Inflation-restriction yields

$$
\begin{aligned}
0 & \rightarrow H^{1}\left(\operatorname{Gal}\left(K^{\prime} / K\left(\zeta_{\ell}\right)\right), \operatorname{ad}^{0}(\bar{\rho}) \operatorname{Gal}\left(E / K^{\prime}\right)\right. \\
& \rightarrow H^{1}\left(\operatorname{Gal}\left(E / K\left(\zeta_{\ell}\right)\right), \operatorname{ad}^{0}(\bar{\rho})\right) \\
& \rightarrow H^{1}\left(\operatorname{Gal}\left(E / K^{\prime}\right), \operatorname{ad}^{0}(\bar{\rho})\right)^{\operatorname{Gal}\left(K^{\prime} / K\left(\zeta_{\ell}\right)\right)} \\
& \rightarrow H^{2}\left(\operatorname{Gal}\left(K^{\prime} / K\left(\zeta_{\ell}\right)\right), \operatorname{ad}^{0}(\bar{\rho})^{\operatorname{Gal}\left(E / K^{\prime}\right)}\right) .
\end{aligned}
$$

The outer terms are zero because $\operatorname{ad}^{0}(\bar{\rho})^{\operatorname{Gal}\left(E / K^{\prime}\right)}=0$. The second term is zero by assumption. Now any $\ell$-group acting on a finite-dimensional non-trivial $\mathbf{F}_{\ell}$ vector space has a non-trivial set of invariants. Since $\operatorname{Gal}\left(K^{\prime} / K\left(\zeta_{\ell}\right)\right) \cong \mathbf{Z} /(\ell)$, this implies $H^{1}\left(\operatorname{Gal}\left(E / K^{\prime}\right), \operatorname{ad}^{0}(\bar{\rho})\right)=0$. Thus (ii) holds over $Y$ instead of $X$.

By induction, we may therefore pass to an extension $Y^{\prime}$ of $X$ over which (i) and (ii) hold, and such that in addition $\bar{\rho}\left(\pi_{1}\left(Y^{\prime}\right)\right)$ has no normal subgroup of index $\ell$. Over $Y^{\prime}$ we can now apply Theorem 3.1, and the result follows. (This uses again the formulation of Conjecture 1.1, which makes it obvious that de Jong's conjecture holds for $\bar{\rho}$, if it holds for $\bar{\rho}_{\mid \pi_{1}\left(Y^{\prime}\right)}$.)

\section{Appendix}

In this paper we use a modified construction of Taylor-Wiles systems introduced in [TW95]. Here we explain this modification in the original context of modular curves of [TW95]. We use the notation of [Dia97] to indicate what the problem is and how we handle it. The main point is that when proving freeness of certain cohomology groups it is enough for the purposes of TW systems to prove this over certain group algebras whose group of characters 'kill torsion'.

A key step in TW systems is to prove, for certain finite set of primes $Q=\left\{q_{1}, \ldots, q_{r}\right\}$ and any positive integer $N$ prime to the primes in $Q$, results towards the freeness of the cohomology group $H^{1}\left(X_{N, Q}, \mathcal{O}\right)_{\mathrm{m}}$ as a module over $\mathcal{O}\left[\Delta_{Q}\right]$ (under the natural action) with $\Delta_{Q}$ the Sylow $\ell$-subgroup (which we may also view as the maximal $\ell$-quotient) of $\prod_{q \in Q}(\mathbf{Z} / q \mathbf{Z})^{*}$, where $\mathrm{m}$ is a $\bmod$ $\ell$ maximal ideal of a certain Hecke algebra which satisfies a certain set of conditions, and where $\mathcal{O}$ is a finite flat extension of $\mathbf{Z}_{\ell}$. Here $X_{N, Q}$ is the modular curve corresponding to the subgroup $\Gamma_{0}(N) \cap \Gamma_{1}\left(q_{1} \cdots q_{r}\right)$. Further the quotient of $H^{1}\left(X_{N, Q}, \mathcal{O}\right)_{\mathrm{m}}$ by the augmentation ideal of $\mathbf{Z}_{\ell}\left[\Delta_{Q}\right]$ is isomorphic to $H^{1}\left(X_{0}(N), \mathcal{O}\right)_{\mathrm{m}}$ if the primes $q_{i}$ in addition satisfy the hypothesis that the mod $\ell$ representation $\bar{\rho}$ corresponding to $\mathrm{m}$ is such that it is unramified at $q_{i}$ and the ratio of the eigenvalues of $\bar{\rho}\left(\right.$ Frob $\left._{q_{i}}\right)$ are not $q_{i}^{ \pm 1}$.

One of the technical steps in proving the freeness is to impose an auxiliary level structure to avoid problems arising from torsion of $\Gamma_{0}(N)$. We indicate an argument that bypasses this.

We observe the following proposition which directly follows from the proof of [Car89, lemme 1].

Proposition A.1. Let $e$ be any integer that kills the torsion of $\Gamma_{0}(N)$. Let $\Delta_{e}^{Q}$ be the subgroup of $\operatorname{Hom}\left(\Delta_{Q}, \mathbf{Q}_{\ell} / \mathbf{Z}_{\ell}\right)$ that consists of eth powers. Consider the twisted sheaf $\mathcal{O}(\chi)$ for any character $\chi \in \Delta_{e}^{Q}$ on $X_{0}\left(N q_{1} \cdots q_{r}\right)$ and assume $\mathcal{O}$ to be large enough to contain all values of $\chi$. Then if $k$ is the residue field of $\mathcal{O}$, the reduction $\mathcal{O}(\chi) \otimes k$ is isomorphic to the constant sheaf $k$ on $X_{0}\left(N q_{1} \cdots q_{r}\right)$. 
As a standard consequence one has the following result.

Corollary A.2. Let $\Delta_{Q}^{e}$ be the quotient of $\Delta_{Q}$ that is dual to the $\operatorname{subgroup} \Delta_{e}^{Q}$ of $\operatorname{Hom}\left(\Delta_{Q}, \mathbf{Q}_{\ell} / \mathbf{Z}_{\ell}\right)$. Let $X_{N, Q, e}$ be the modular curve that corresponds to the congruence subgroup that is the kernel of the natural map $\Gamma_{0}\left(N q_{1} \cdots q_{r}\right) \rightarrow \Delta_{Q}^{e}$. Let $\mathrm{m}$ be a maximal ideal of a certain Hecke algebra (as in [Dia97]: we drop operators $T_{r}, U_{r}$ for $r$ not coprime to $\left.N q_{1} \cdots q_{n}\right)$ acting on $H^{1}\left(X_{N, Q, e}, \mathcal{O}\right)$ such that the corresponding residual representation $\bar{\rho}$ of $G_{\mathbf{Q}}$ is not reducible. Then $H^{1}\left(X_{N, Q, e}, \mathcal{O}\right)_{\mathrm{m}}$ has a natural action of $\Delta_{Q}^{e}$ and is a free $\mathcal{O}\left[\Delta_{Q}^{e}\right]$-module for any $\mathcal{O}$ that is finite flat over $\mathbf{Z}_{\ell}$.

Proof of Proposition A.1. For conciseness of notation we denote by $Y$ and $X$ the curves $X_{N, Q, e}$ and $X_{0}\left(N q_{1} \cdots q_{r}\right)$, and we have the natural map $r: Y \rightarrow X$ that is the quotient by $\Delta_{Q}^{e}$. The sheaf $\mathcal{O}(\chi)$ is described as $\Delta_{Q}^{e} \backslash[Y \times \mathcal{O}]$ with $\Delta_{Q}^{e}$ acting on the constant sheaf $\mathcal{O}$ by $\chi$. The stalk at a point $x \in X$, after choosing a point $y$ in $r^{-1}(x)$, can be identified with the subset of the stalk at $y$ of the constant sheaf, $\mathcal{O}_{y}$, on which the stabilizer of $y$ in $\Delta_{Q}^{e}$ acts by $\chi$ (thus it is either $\mathcal{O}_{y}$ or 0 ). From this description the proposition follows using our assumption on $\chi$.

Proof of Corollary A.2. We first note that as $\bar{\rho}$ is irreducible, the étale $H^{0}$ and $H^{2}$ of modular curves with coefficients in the twisted sheaves above do not have the maximal ideal $\mathrm{m}$ in their support (see [Car89, §3]). Thus from the proposition above, and the long exact sequence of cohomology, it follows that for any character $\chi \in \Delta_{e}^{Q}$ on $X_{0}\left(N q_{1} \cdots q_{r}\right)$ we have a (Hecke equivariant) isomorphism $H^{1}\left(X_{0}\left(N q_{1} \cdots q_{r}\right), \mathcal{O}(\chi)\right)_{\mathrm{m}} \otimes k \simeq H^{1}\left(X_{0}\left(N q_{1} \cdots q_{r}\right), k\right)_{\mathrm{m}}$. Then by a standard argument (see proof of Proposition 5.6.1 in [CDT99]) the corollary follows.

Let us further assume that for each $n \in \mathbf{N}$ we have sets of primes $Q_{n}$ of constant cardinality $r$ such that, for $q \in Q_{n}, q$ is prime to $N, q$ is $1 \bmod \ell^{n}$, and $\bar{\rho}$ is unramified at $q$ with the ratio of the eigenvalues of $\rho\left(\right.$ Frob $\left._{q}\right)$ not $q^{ \pm 1}$ (such sets exist when $\bar{\rho}$ restricted to the quadratic subfield of $\mathbf{Q}\left(\mu_{\ell}\right)$ is not reducible). Then again $H^{1}\left(X_{N, Q, e}, \mathcal{O}\right)_{\mathrm{m}}$ is a free $\mathcal{O}\left[\Delta_{Q_{n}}^{e}\right]$-module whose quotient by $\Delta_{Q_{n}}^{e}$ is isomorphic to $H^{1}\left(X_{0}(N), \mathcal{O}\right)_{\mathrm{m}}$. The group $\Delta_{Q_{n}}^{e}$ surjects onto $\mathbf{Z} /\left(\ell^{n-e}\right)^{r}$, and thus grows systematically with $n$. This is enough to construct TW systems as in [Dia97, §3.1] avoiding imposition of auxiliary level structures.

The modification of this appendix can also be used to avoid imposition of auxiliary level structure needed to bypass torsion problems in the level lowering method of [SW01], as done in the main body of this paper. Here the further remark, in addition to the observation above, is that when base changing to make orders of multiplicative groups of orders of residue fields at some fixed places congruent to 1 modulo high powers of $\ell$, one also requires certain other places, chosen in advance, to split completely, so that the $\ell$-part of the torsion cannot grow much under base change.

\section{ACKnowledgements}

GB would like to thank the TIFR, Mumbai, for its hospitality in the summer of 2002 during which the first decisive steps in this work were taken, and also the ETH Zürich for its inspirational environment and for its generosity in providing a three-year post-doctoral position.

Some of CK's work on this paper was done during a visit to Université Paris 7 which was supported by the Centre franco-indien pour la promotion de la recherche avancée (CEFIPRA) under Project 2501-1 'Algebraic Groups in Arithmetic and Geometry'.

\section{References}

Böc03 G. Böckle, Lifting mod $p$ representations to characteristic $p^{2}$, J. Number Theory 101 (2003), 310-337. 


\section{MOD $\ell$ REPRESENTATIONS OF ARITHMETIC FUNDAMENTAL GROUPS, II}

BK05 G. Böckle and C. Khare, Mod $\ell$ representations of arithmetic fundamental groups, I: An analog of Serre's conjecture for function fields, Duke Math. J. 129 (2005), 337-369.

BK03 G. Böckle and G. Khare, Preprint (2003), http://xxx.lanl.gov/abs/math.NT/0312490.

BK06 G. Böckle and C. Khare, Finiteness results for mod $\ell$ Galois representations over function fields, Preprint (2006).

BJ79 A. Borel and H. Jacquet, Automorphic forms and automorphic representations, in Proc. Symp. Pure Mathematics XXXIII, Part 1 (American Mathematical Society, Providence, RI, 1979), 189-207.

Car89 H. Carayol, Sur les représentations galoisiennes modulo $\ell$ attachées aux formes modulaires, Duke Math. J. 59 (1989), 785-801.

Car94 H. Carayol, Formes modulaires et représentations galoisiennes à valeurs dans un anneau local complet, in p-adic monodromy and the Birch and Swinnerton-Dyer conjecture, Boston, MA, 1991, Contemporary Mathematics, vol. 165 (American Mathematical Society, Providence, RI, 1994), 213-237.

CPS75 E. Cline, B. Parshall and L. Scott, Cohomology of finite groups of Lie type I, Publ. Math. Inst. Hautes Études Sci. 45 (1975), 169-191.

CDT99 B. Conrad, F. Diamond and R. Taylor, Modularity of certain potentially Barsotti-Tate Galois representations, J. Amer. Math. Soc. 12 (1999), 521-567.

Dia97 F. Diamond, The Taylor-Wiles construction and multiplicity one, Invent. Math. 128 (1997), 379-391.

Dri83 V. G. Drinfeld, Two-dimensional $\ell$-adic representations of the fundamental group of a curve over a finite field and automorphic forms on GL(2), Amer. J. Math. 105 (1983), 85-114.

Fuj K. Fujiwara, Deformation rings and Hecke algebras in the totally real case, unpublished manuscript.

Gai04 D. Gaitsgory, On de Jong's conjecture, Preprint (2004), http://arXiv.org/abs/math/0402184.

HT03 M. Harris and R. Taylor, Deformations of automorphic Galois representations, Draft (2003), http://www.math.jussieu.fr/ harris/.

dJo01 A. J. de Jong, Conjecture on arithmetic fundamental groups, Israel J. Math. 121 (2001), 61-84.

Kha00 C. Khare, Conjectures on finiteness of mod $p$ Galois representations, J. Ramanujan Math. Soc. 15 (2000), 23-42.

Laf02 L. Lafforgue, Chtoucas de Drinfeld et correspondance de Langlands, Invent. Math. 147 (2002), $1-241$.

Maz89 B. Mazur, Deforming Galois representations, in Galois groups over Q, Mathematical Sciences Research Institute Publications, vol. 16 (Springer, New York, 1989), 385-437.

Moo00 H. Moon, Finiteness results on certain mod $p$ Galois representations, J. Number Theory 84 (2000), $156-165$.

MT01 H. Moon and Y. Taguchi, Mod p Galois representations of solvable image, Proc. Amer. Math. Soc. 129 (2001), 2529-2534.

Ogi05 D. Ogilvie, Lifting mod $\ell$ cusp forms, Preprint (2005).

SW01 C. M. Skinner and A. J. Wiles, Base change and a problem of Serre, Duke Math. J. 107 (2001), $15-25$.

TW95 R. Taylor and A. Wiles, Ring-theoretic properties of certain Hecke algebras, Ann. of Math. (2) 141 (1995), 553-572.

Wil95 A. Wiles, Modular elliptic curves and Fermat's last theorem, Ann. of Math. (2) 141 (1995), 443-551.

Gebhard Böckle boeckle@iem.uni-due.de

Institut für experimentelle Mathematik, Universität Duisburg-Essen, Standort Essen,

Ellernstrasse 29, 45326 Essen, Germany

Chandrashekhar Khare shekhar@math.utah.edu, shekhar@math.tifr.res.in

Department of Mathematics, University of Utah, 155 S 1400 E, Salt Lake City, UT 84112, USA

Current address: School of Mathematics, TIFR, Homi Bhabha Road, Mumbai 400 005, India 